Portland State University

PDXScholar

7-14-2020

\title{
Droplet Ejections During Wet Lab Operations Aboard Spacecraft
}

\author{
Caleb Cushman Turner \\ Portland State University
}

Follow this and additional works at: https://pdxscholar.library.pdx.edu/open_access_etds

Part of the Aerodynamics and Fluid Mechanics Commons, and the Mechanical Engineering Commons Let us know how access to this document benefits you.

\section{Recommended Citation}

Turner, Caleb Cushman, "Droplet Ejections During Wet Lab Operations Aboard Spacecraft" (2020). Dissertations and Theses. Paper 5549.

https://doi.org/10.15760/etd.7423

This Thesis is brought to you for free and open access. It has been accepted for inclusion in Dissertations and Theses by an authorized administrator of PDXScholar. Please contact us if we can make this document more accessible: pdxscholar@pdx.edu. 
Droplet Ejections During Wet Lab Operations

Aboard Spacecraft

\author{
by \\ Caleb Cushman Turner
}

A thesis submitted in partial fulfillment of the requirements for the degree of

\author{
Master of Science \\ in \\ Mechanical Engineering
}

Thesis Committee:

Mark Weislogel, Chair

Derek Trethaway

Raúl Buyoán Cal

Portland State University

2020 


\begin{abstract}
The breakup and rupture of liquid bridges, thin films, bubbles, droplets, rivulets, and jets can produce satellite droplets that are subsequently ejected into their surrounding environment. For example, when any solid object is withdrawn from a liquid bath, the formation of an ever-thinning columnar liquid bridge eventually ruptures along the axis of the bridge. When rupture occurs under typical pipetting conditions the dynamics governing the rupture almost always produce at a minimum a satellite droplet. When these droplets occur they are often too small and too fast to be observed by the human eye. In a terrestrial environment they are of little concern due to the gravitational force imperceptibly returning these droplets back to the bulk fluid. This is not the case for low- $g$ environments where activities such as pipetting creates satellite droplets that are ejected far away from the source fluid creating a risk of contamination within the surrounding working environments. In this work we demonstrate a variety of droplet ejections for the application of pipetting in space and highlight how in a low- $g$ environment such dynamics depend on system geometries, fluid properties, wettability, and withdrawal rate. A drop tower data set is collected in support of a regime map organized by withdrawal Weber and Capillary numbers that highlight when different fluid ejection types are to be expected. Mitigation techniques are presented as a design guide for further applications aboard spacecraft.
\end{abstract}




\section{Acknowledgements}

The author would like to thank the entire DDT lab past and present: Thank

you Karl, Logan, Rawand, Jesse, Rihana, and Sam. Thank you to the NASA collaborators Gene Ungar and Jennifer Buchli. Thank you to the astronauts Alex Gerst, Peggy Winston, Kate Rubins, Scott Kelly, Kjell Lindgren, Luca Parmitano, and Christina Koch. Thank you to my wife for her tireless support. Finally, thank you to Dr. Mark Weislogel for the years of mentorship.

This work was supported by NASA Cooperative Agreements 80NSSC18K0436 and 80NSSC18K0161 


\section{Contents}

Abstract $\quad$ i

Acknowledgements

List of Tables $\quad$ v

List of Figures vi vi vis

Nomenclature xiii

1 Introduction and Motivation 1

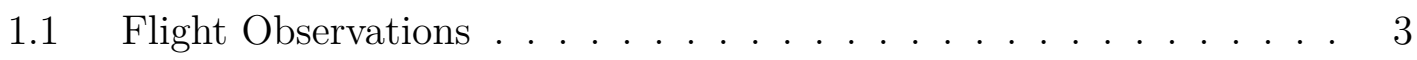

2 Theory $\quad 8$

3 Experiments $\quad 14$

4 Numerical Analysis $\quad 18$

4.1 Liquid Bridge Statics for Pipetting Systems . . . . . . . . . . . . 18

4.2 Liquid Bridge Dynamics For Pipetting Systems . . . . . . . . . . 20

5 Results $\quad 23$ 


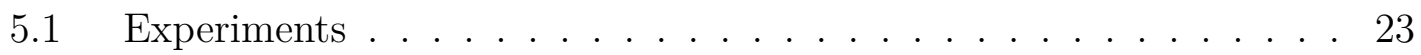

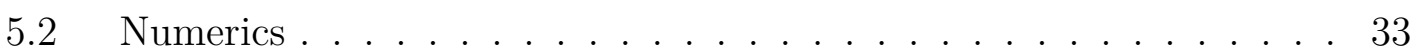

5.3 Pipette Angle Effects . . . . . . . . . . . . . . . . . 37

6 Mitigation Strategies $\quad 40$

6.1 Introduction ........................ 40

6.2 Deep Well Container Geometry . . . . . . . . . . . . . . . 41

6.3 Air Flow Control . . . . . . . . . . . . . . . . . 44

6.4 Superhydrophobic Instrument Exterior . . . . . . . . . . . . . . 46

7 Summary \& Future Work $\quad 48$

$\begin{array}{ll}\text { Bibliography } & 52\end{array}$

$\begin{array}{lr}\text { Appendices } & 56\end{array}$

Appendix A: Experimental Parameters . . . . . . . . . . . . 56

Appendix B: Governing Equations . . . . . . . . . . . . . . 65 
List of Tables

3.1 Fluid properties at room temperature . . . . . . . . . . . 17 


\section{List of Figures}

1.1 Sketches depicting the time evolution of pipette-withdrawal induced liquid bridge a. formation b. elongation c. rupture, and satellite droplet ejection. . . . . . . . . . . . . . . . .

1.2 Image sequence $(\sim 7 \mathrm{~Hz})$ from STCE on ISS showing $\sim 1 \mathrm{~mm}$ diameter ejected satellite droplet produced via routine pipetting withdrawal. Water with green dye for contrast is used as the working fluid. A crew comfort fan covered by a red cotton cloth provides containment airflow suction into the cloth (right to left) to capture ejected droplets. The ejected droplet is entrained by the air flow with larger droplets readily absorbed by the cloth on impact. . . . 5 
1.3 Slime in Space demonstration with astronauts observing manually separated viscous liquid bridges formed between $13.34 \mathrm{~cm}$ diameter 'Ping Pong' paddles. a. Paddle separation velocity $U_{o}=0.005$ $\mathrm{m} / \mathrm{s}$ is below a critical limit in which rupture occurs at one axial location and no satellite droplets are observed. b. Higher separation velocity $U_{o}=0.29 \mathrm{~m} / \mathrm{s}$ with no satellite droplets observed upon rupture. c. One single $\sim 28 \mathrm{~mm}$ diameter droplet is observed upon bridge rupture, for separation velocity $U_{o}=0.5 \mathrm{~m} / \mathrm{s}$ nearly twice that of b. d. Higher velocity separation $U_{o}=0.8 \mathrm{~m} / \mathrm{s}$ creates unearthly-sized liquid bridge, where rupture occurs at multiple locations along axis and creates multiple visible droplets, $\sim 30 \mathrm{~mm}$

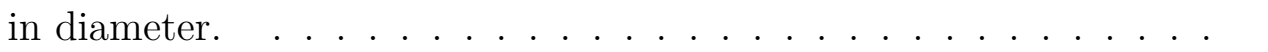

2.1 Schematic of the pipette liquid-bridge system. . . . . . . . . . . . 9

3.1 Dryden drop tower and experiment rig schematic: a. experiment rig with support equipment b. drag shield c. tower and elevation schematic. . . . . . . . . . . . . . . 15

3.2 CAD schematic of experiment rig assembly. Breakout view of the orientation and nomenclature for the imaging, lighting, and fluid withdrawal systems. Not shown are the Arduino controller circuit and assorted camera/power cables. . . . . . . . . . . 16 
3.3 a. Cannula geometries employed with $r_{o}=0.5,1.8$, and $2 \mathrm{~mm}$ respectively b. Isometric CAD view of container geometries employed $R=10,5,3,2,1 \mathrm{~mm}$ respectively. All containers maintained the same aspect ratio $h_{r e s} / R=3 \ldots \ldots \ldots$. . . . . . . . . 16

4.1 Numerically computed free surface profiles for the pipette pre-built model. a. initial condition, b. rough convergence, and c. deeply refined converged solution . . . . . . . . . . . . . . . 19

4.2 Example dynamic meshing in Gerris using the AMR algorithm highlighting increased mesh density at areas with large curvature. The time sequence highlights meshing of the interface at $t=0 \mathrm{~s}$, during liquid bridge necking, and when the bridge ruptures and a droplet is ejected. Dimensionless parameters defining this simulation are $\mathrm{We}_{o}^{1 / 2}=0.37$ and $\mathrm{Ca}_{o}=0.0012 \ldots . . . . . .21$

$5.1 \quad$ Three Distinct liquid ejection regimes observed during low- $g$ experiments. a. 'Satellite' droplet, $\delta=0.06$ (image sequence at $105 \mathrm{~Hz}$ ) b. 'Mother' droplet, $\delta=0.6$ (image sequence at $770 \mathrm{~Hz}$ ) and c. 'Ligament ejection', $\delta=1.05$ (image sequence at $150 \mathrm{~Hz}$ ) . . . . . 26

5.2 Regime maps for droplet ejections in a. low- $g$ and b. $1-g_{o}$. Large volume ligament ejections appear to be exclusive to low- $g$ environments where $g$ induced drain does not act to thin these bridges while elongation occurs. . . . . . . . . . . . . . . . 27 
5.3 Approximately constant $\mathrm{We}_{o}^{1 / 2} \sim 1.3$ withdrawals for $1 \mathrm{~mm}$ OD instrument (scale factor is constant for all images) with increasing $\mathrm{Ca}_{o}$ via increasing viscosity a. $\mathrm{Ca}_{o} \sim 0.006$ b. $\mathrm{Ca}_{o} \sim 0.007$ and c. $\mathrm{Ca}_{o} \sim 0.07$. Image sequence highlights critical dynamic bridge heights and ejected droplet sizes increase with viscosity. . . . . . . 28

5.4 Approximate regime boundary transitions in low- $g$ between satellite to mother droplet ejections and mother-ligament droplet ejections as observed experimentally for the three distinct fluid regimes determined by $\mathrm{Su}^{1 / 2}$. Note that ligament* is an assumed ligament regime. Equivalent droplet radii from the Slime in Space demonstration used to calculate corresponding $\delta$ 's are small due to the large boundary geometry from the ping pong paddles used to separate these liquid bridges. . . . . . . . . . . . . . . . . 30

5.5 a. Regime map of Fig. 5.2 with the 'Slime in Space' demonstration experiments included. b. Unearthly sized liquid ligament stretched beyond stable limits in low- $g$ leading to breakup producing multiple droplets. c. Slower retraction breakup with Slime where only a single droplet is produced with ligaments returning back to the bulk fluid on paddles before further rupture can occur. d. Slow quasi-steady Slime bridge rupture where no satellite droplets are observed. . . . . . . . . . . . . . . . 31 
5.6 Marginal stability curves computed by SE-FIT for a range of $\mathrm{Bo}_{\text {unit }}$. Quasi-steady experimental bridge heights are included for comparison. Selected images of the liquid bridge marginal stable configurations are included above plot to highlight asymmetric configurations for all $\mathrm{Bo}_{\text {unit }}$ when $r_{o} / R>0.5 . \ldots . . . . . . . . .34$

5.7 Numerically calculated regime map for low- $g$ pipette withdrawals using the open source code Gerris. Regime transitions for the inertial-capillary balance are approximately $\mathrm{We}_{o}^{1 / 2} \sim 1$ for the satellite to mother droplet transition and $\mathrm{We}_{o}^{1 / 2} \sim 10$ for the mother droplet to ligament ejection transition. . . . . . . . . . . . 36

5.8 A low- $g$ experiment vs. Gerris run for $45.78 \% \mathrm{H}_{2} \mathrm{O} / 54.22 \%$ Glycerol mixture, instrument outer diameter is $3.8 \mathrm{~mm}$ with a relative retraction velocity of $U_{o}=0.34 \mathrm{~m} / \mathrm{s}, \mathrm{Ca}_{o} \sim 0.07$ and $\mathrm{We}_{o}^{1 / 2} \sim 2.5$. The experimental value of $\delta=0.68$ while the computed value is

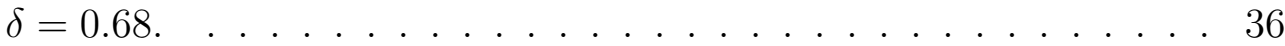

5.9 Bridge rupture and droplet production for pipette retractions at varying angles, $\phi$ : a. initial wetting condition for each instrument, b. liquid bridge critical height just prior to breakup, and c. ejected droplets produced. A noticeable increase in ejected droplet volume

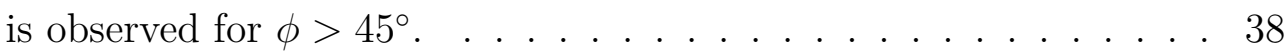

5.10 Schematic showing the new governing geometry for these large instrument tilt withdrawals. . . . . . . . . . . . . . 38 
5.11 The ratio $\delta=r_{\text {drop }} / r_{o}$ as a function of $\phi$. For $\phi \gtrsim 45^{\circ}$ we observe a transition from 'mother' droplets to large ligament ejections with a substantial increase in ejected fluid volume. . . . . . . . . . . . 39

6.1 Selection of composite images highlighting ejected droplet trajectories: a. direct droplet ejection where the droplet is generated between the instrument and reservoir boundaries with some unknown ejection angle, here $\alpha \sim 60^{\circ}$ from the withdrawal direction. b. ejection and rebound, where the droplet ejects towards the bath and rebounds off the free surface escaping the container, and c. droplet ejected directly into the reservoir free surface, where the droplet re-coalesces back into the bulk fluid. . . . . . . . . . . . . 41

6.2 Pipette container schematic for deep well mitigation. . . . . . . . . 42

6.3 Dimensionless fill fraction as a function of mitigation angle $\alpha$ for containers of varying aspect ratios $R / h_{\text {res }}$. . . . . . . . . . . 43

6.4 Airflow control schematic. A droplet is introduced into a free stream airflow in the $x$-direction. The droplet has an assumed initial velocity component in only the $y$-direction, $v_{o}$. The airflow is confined to some area based on a height in the $y$-direction and some width into the plane. . . . . . . . . . . . . . . . 44 
6.5 a. Eq. 6.4 and 6.5 model compared with data for experiment of ejected droplet in time. b. Composite image from experiment highlighting the effect of airflow containment on droplet trajectories. Droplet initial velocity is assumed straight upward with a magnitude approximately that of $U_{o} \sim 0.5 \mathrm{~m} / \mathrm{s}$. Positioned to the left, out of frame are two vertically stacked $40 \mathrm{~mm}^{2}$ electronics cooling fans blowing air at $\sim 6.9 \mathrm{CFM}\left(U_{\infty} \sim 2.5 \mathrm{~m} / \mathrm{s}\right)$ horizontally rightward. ..................... . . 4

6.6 STAR-CCM+ Superhyrophobic instrument tip $\left(r_{o}=0.5 \mathrm{~mm}\right.$, $\left.r_{i}=0.25 \mathrm{~mm}\right)$ simulation with $\theta_{\text {exterior }} \sim 150^{\circ}$ and $\theta_{\text {interior }} \sim 30^{\circ}$ prescribed drain velocity is $0.5 \mathrm{~m} / \mathrm{s}$. Immediate differences between SHS tip and conventional exteriors is the change in liquid bridge upper boundary conditions. The characteristic dimension becomes the instrument inner radii $r_{i}$ rather than $r_{o}$. . . . . . . . . . 47 


\section{Nomenclature}

\section{Latin Letters}

$\begin{array}{ll}A_{a} & \text { Airflow area } \\ \mathrm{Bo}_{\text {unit }} & \text { Unit Bond number }=\rho g / \sigma\left(\mathrm{m}^{-2}\right) \\ d_{\text {drop }} & \text { Diameter of ejected droplet } \\ F_{\text {air }} & \text { Force of free stream airfield } \\ g & \text { Acceleration field strength }\left(\mathrm{m} / \mathrm{s}^{2}\right) \\ g_{o} & \text { Terrestrial gravitational acceleration }\left(9.81 \mathrm{~m} / \mathrm{s}^{2}\right) \\ h_{b} & \text { Height of the liquid bridge at rupture } \\ h_{r e s} & \text { Height of fluid reservoir } \\ h_{f i l l}^{*} & \text { Dimensionless container fill height } \\ r & \text { Radial coordinate } \\ r_{i} & \text { Instrument tip inner radius } \\ r_{o} & \text { Instrument tip outer radius } \\ r_{o}^{+} & \text {Modified instrument tip outer radius } \\ R & \text { Container inner radius } \\ t_{\sigma} & \text { Capillary time scale }=\left(\rho r^{3} / \sigma\right)^{1 / 2} \\ U_{o} & \text { Instrument withdrawal velocity }(\mathrm{m} / \mathrm{s}) \\ U_{r} & \text { Radial component of velocity }(\mathrm{m} / \mathrm{s}) \\ U_{s} & \text { Velocity scale }(\mathrm{m} / \mathrm{s}) \\ U_{z} & \text { Zed component of velocity }(\mathrm{m} / \mathrm{s}) \\ U_{\infty} & \text { Free stream airflow velocity } \\ x & \text { x coordinate } \\ y & \text { y coordinate } \\ z & \text { z coordinate }\end{array}$




\section{Greek Letters}

$\begin{array}{ll}\alpha & \text { Mitigation angle } \\ \beta & \text { Airflow control constant } \\ \delta & \text { Ejected droplet aspect ratio } r_{d r o p} / r_{o} \\ \dot{\epsilon} & \text { Strain rate }(1 / \mathrm{s}) \\ \phi & \text { Instrument retraction angle } \\ \gamma & \text { Liquid bridge slenderness aspect ratio } r_{o} / h_{b} \\ \mu & \text { Dynamic viscosity of fluid }(\mathrm{kg} /(\mathrm{m} \cdot \mathrm{s})) \\ \mu_{a} & \text { Dynamic viscosity of air }(\mathrm{kg} /(\mathrm{m} \cdot \mathrm{s})) \\ \rho & \text { Density of fluid }\left(\mathrm{kg} / \mathrm{m}^{3}\right) \\ \rho_{a} & \text { Density of air }\left(\mathrm{kg} / \mathrm{m}^{3}\right) \\ \sigma & \text { Surface tension }\left(\mathrm{N} / \mathrm{m}^{2}\right) \\ \theta & \text { Contact angle }\end{array}$

\section{Dimensionless Groups}

$\begin{array}{ll}\mathrm{Bo}_{o} & \text { Bond number of instrument }=\rho g r_{o}^{2} / \sigma \\ \mathrm{Ca}_{o} & \text { Capillary number of instrument }=\mu U_{o} / \sigma \\ \mathrm{Fr} & \text { Froude number of instrument withdrawal }=U_{o} /\left(g r_{o}\right)^{1 / 2} \\ \mathrm{Oh} & \text { Ohnesorge number }=\mu /\left(\rho r_{o} \sigma\right)^{1 / 2} \\ \mathrm{Re}_{o} & \text { Reynolds number of instrument }=\rho U_{o} r_{o} / \mu \\ \mathrm{Su} & \text { Suratman number }=\rho r_{o} \sigma / \mu^{2} \\ \mathrm{We}_{o} & \text { Weber number of instrument }=\rho r_{o} U_{o}^{2} / \sigma\end{array}$




\section{Acronyms}

$\begin{array}{ll}\text { AMR } & \text { Adaptive mesh refinement } \\ \text { CAD } & \text { Computer aided design } \\ \text { CFD } & \text { Computational fluid dynamics } \\ \text { CFM } & \text { Cubic feet per minute } \\ \text { DC } & \text { Direct current } \\ \text { FIJI } & \text { FIJI is just image J } \\ \text { fps } & \text { frames per second } \\ \text { GUI } & \text { Graphical user interface } \\ \text { HD } & \text { High definition } \\ \text { HF } & \text { Height factor } \\ \text { ISS } & \text { International space station } \\ \text { LED } & \text { Light emitting diode } \\ \text { NASA } & \text { National Aeronautical and Space Administration } \\ \text { SE-FIT } & \text { Surface evolver fluid interface tool } \\ \text { SLA } & \text { Stereo lithography } \\ \text { STCE } & \text { Surface tension containment experiment } \\ \text { VOF } & \text { Volume of fluid }\end{array}$




\section{Chapter 1}

\section{Introduction and Motivation}

Liquid volume breakup is a universal phenomena arising in nature and numerous industrial processes. Perhaps the first mathematical study of these free surface flows began with work published by Lord Rayleigh in the latter part of the 19th century concerning the linear stability of liquid jets. During the next century research into these types of free surface flows only extended Rayleigh's work in linear stability theory through considerations of variable perturbations, fluid geometries, improved resolution, etc. For example, Tomotika [1] accounted for the surrounding fluid and Keller et al. [2] examined a progressive wave disturbance to the fluid volume that grew along the axial coordinate but remained constant in time. More recently there has been extensive focus on the dynamics of unstable axisymmetric capillary bridges of a known volume carefuly stretched to the point of initiating rupture, though the role of inertia between the bridge boundary conditions has been of little concern. To our knowledge, most work is carried out well below the Stokes limit. The literature is focused on the interfacial dynamics of the unstable approach to the finite time singularity of a discontinuity in the fluid volume. An abundance of experiments on capillary bridge dynamics conducted in Plateau tanks is well documented which highlights these interfacial dynamics in a reduced buoyant force 
environment [3], as well as Meseguer et al.'s [4] work on stable bridge configurations aboard the Space Shuttle Spacelab D1 mission. What remains ambiguous in previous studies is the combination of inertial effects defined at the bridge boundaries, such as axial velocity normal to the fluid interface and the resultant production of satellite and mother droplets upon rupture, as illustrated in Fig. 1.1. Tjahjadi et al. [5] provide substantial information concerning the production of satellite and mother droplets upon capillary breakup within a Couette device and show how under certain circumstances, namely the viscosity ratio of the working fluid to its host fluid, the phenomena of droplet production may be an ever shrinking, self-repeating process. Dodds et al. [6] provide an in-depth analysis concerning the role of inertia and droplet volume deposition on a moving substrate directed axially away from the fluid reservoir. The results observed from terrestrial experiments with large strain rates $\left(\dot{\epsilon} t_{\sigma} \gg 1\right)$ for liquid ligament elongation have been shown to increase the volume of ejected fluid as an array of multiple droplets of varying sizes [7].

Apart from such work, the reports on satellite droplet production via axial separation is only a briefly noted consequence of the rupture dynamics for these axisymmetric liquid bridges. Herein we seek to quantify the roles of inertia, surface tension, viscosity, and system geometry on satellite droplet production. We are most concerned with the unique low- $g$ impacts during wet lab unit operations aboard spacecraft, where mitigation techniques must begin with knowledge of satellite droplet volumes, trajectories, and velocities. 

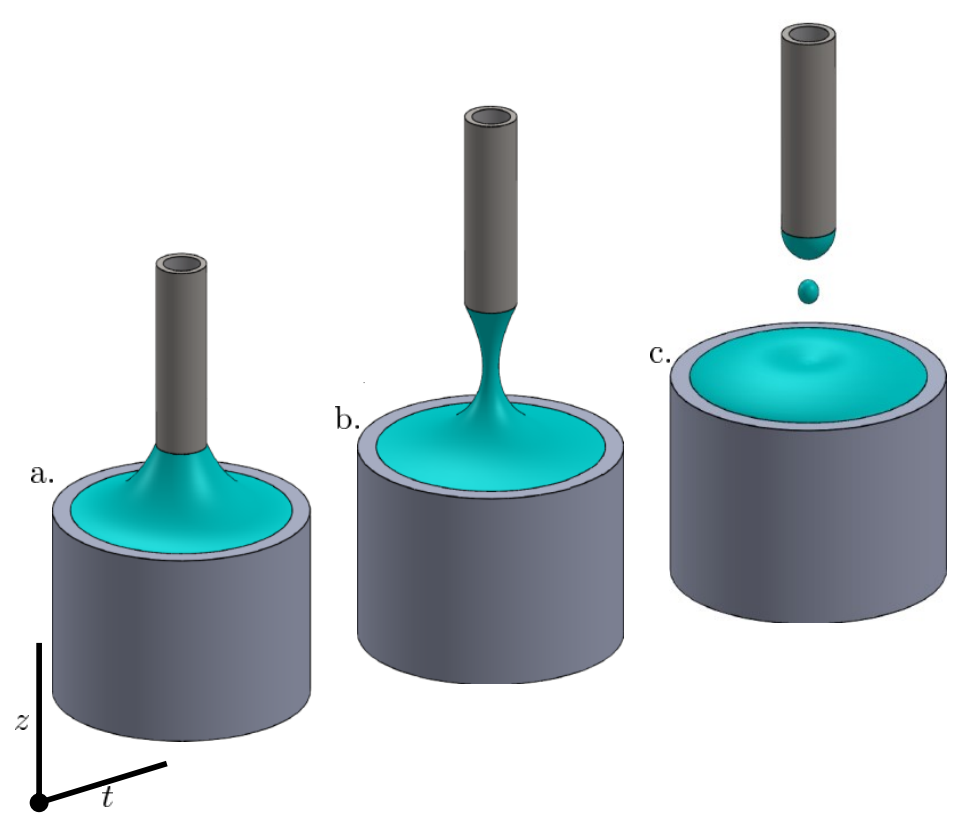

Figure 1.1: Sketches depicting the time evolution of pipette-withdrawal induced liquid bridge a. formation b. elongation c. rupture, and satellite droplet ejection.

\section{$1.1 \quad$ Flight Observations}

The Capillary Beverage Experiment [8] conducted aboard the ISS may have caused a fundamental shift in how NASA views the handling of zero-toxicity fluids in the open cabin of the ISS. The demonstration showed that fluids may be routinley passively controlled via surface tension and system geometry alone, opening up more avenues of scientific throughput by allowing experiments to be conducted in the open cabin rather than the more cumbersome and restricting Micro gravity Science Glove box (MSG). Following the Capillary Beverage demonstration, astronaut Kate Rubins conducted the Biomolecule Sequencer Investigation [9]. During these activ- 
ities routine pippetting operations were performed to sequence DNA on ISS for the first time aboard a spacecraft. Even with numerous repetitive insertions and withdrawals of a pipette within fluid containers (wells), there were no satellite droplets reported by the crew because they were not observed, not necessarily because they were not present. NASA continued to asses the possibility of ejected droplets upon liquid bridge rupture with dynamics similar in nature to the pipetting operations of the Biomolecule Sequencer Investigation. We highlight the recent ISS results of the Surface Tension Containment Experiment (STCE) [10], which sought to identify situations that produce such droplets and how simple air flows may be employed to capture them. The experiments employ pipette and liquid well geometries, a selection of which are shown in Fig. 1.2. During the STCE operations both astronauts and ground teams did not observe satellite droplets for hundreds of pipette withdrawal operations. However, a post-flight assessment of the video with enhanced contrast, decreased playback speed, and increased magnification indeed revealed numerous ejected satellite droplets leaving the well plate reservoir upon bridge rupture with an example ejection highlighted in Fig. 1.2. We note that for STCE the image resolution, frame rate (30 fps), and camera working distance were at approximately human eye level, and not able to adequately capture the wide majority of the fast $(\leq 1 \mathrm{~m} / \mathrm{s})$ submillimetric droplets formed during the routine manual pipetting operations. Low- $g$ drop tower tests reported herein employ increased magnification and frame rate which more clearly and consistently reveal the phenomena.

Additional observations include the joint NASA-Nickelodeon ISS STEM flight experiment [11], 'Slime in Space' demonstrations. We note that Slime is a weakly visco-elastic fluid with viscosity approximatley 20,000 times that of water $(\mu \sim 200$ 


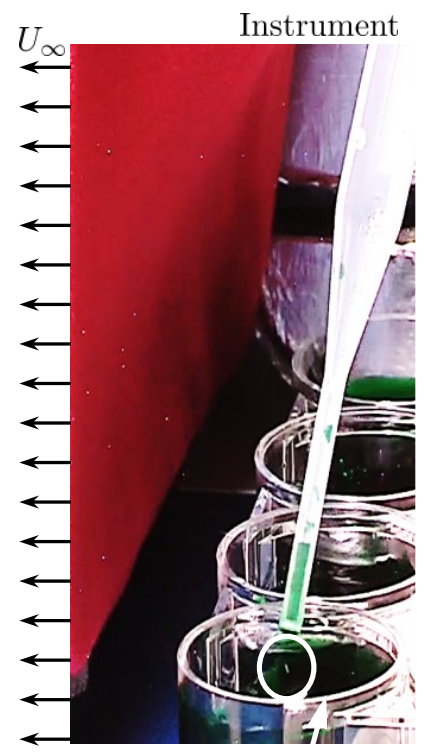

Crew cloth

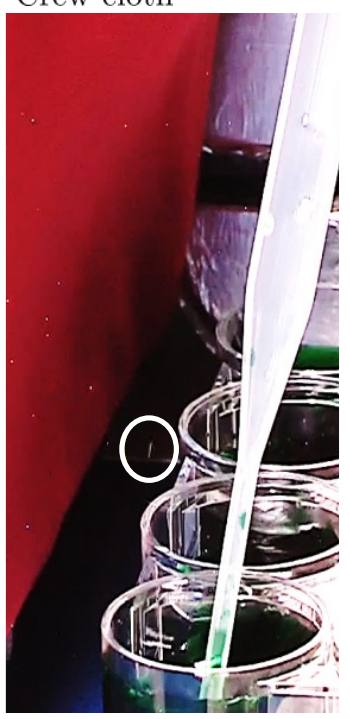

Ejected satellite droplet

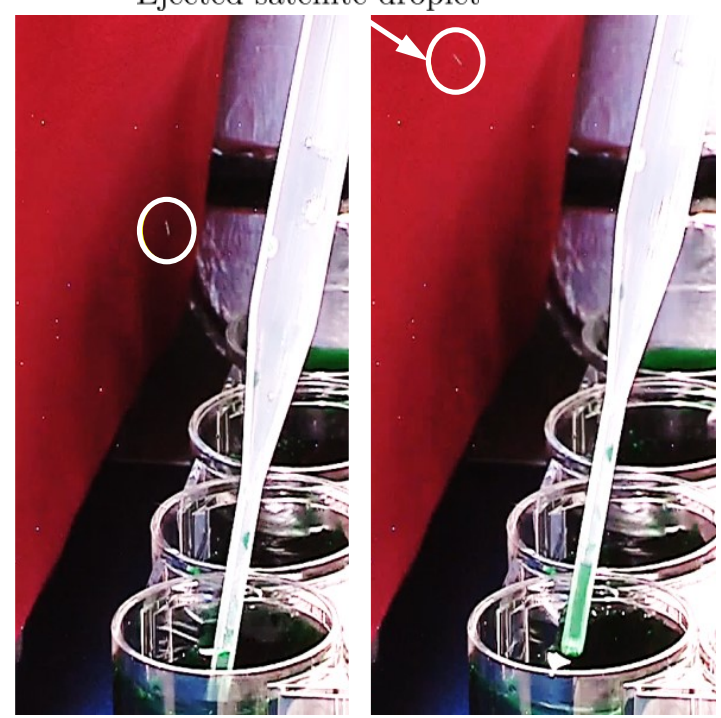

Liquid well/reservoir

Figure 1.2: Image sequence $(\sim 7 \mathrm{~Hz})$ from STCE on ISS showing $\sim 1 \mathrm{~mm}$ diameter ejected satellite droplet produced via routine pipetting withdrawal. Water with green dye for contrast is used as the working fluid. A crew comfort fan covered by a red cotton cloth provides containment airflow suction into the cloth (right to left) to capture ejected droplets. The ejected droplet is entrained by the air flow with larger droplets readily absorbed by the cloth on impact.

$\mathrm{kg} /(\mathrm{m} \cdot \mathrm{s}))$. Among a number of crew interactions with Slime in the open cabin of the ISS, astronauts also conducted demonstrations of liquid bridge rupture. An example of nearly static bridge rupture is shown in Fig. 1.3a, where the visco-capillary balance leads to no satellite ejections observed. At intermediate retraction velocities the liquid bridge elongates significantly but does not produce visible satellites as shown in Fig. 1.3b. Observations of steady intermediate withdrawal rates in Fig. 1.3a and b reveal results similar to Smolka [12] who report no satellite droplets produced when liquid rupture occurs during dripping of a micellar non-Newtonian fluid from a $1.54 \mathrm{~mm}$ inner radius circular orifice. However in Fig. $1.3 \mathrm{c} \& \mathrm{~d}$, at sufficient velocities $U_{o} \sim 0.8 \mathrm{~m} / \mathrm{s}$, an unearthly bridge is established that subsequently breaks- 
up forming multiple droplets, roughly $30.5 \mathrm{~mm}$ in diameter.

It is the hope of the author to provide engineers, designers, and scientists with more clarity into the highly variable phenomena of ejected droplets from liquid bridge rupture, specifically for the activity of pipetting aboard spacecraft. Presented in the following chapters are dimensionless groups highlighting different types of droplet ejections and three different ejection trajectory types that may arise in low$g$ environments. The information contained herein should allow for designers and researchers to anticipate, control, contain, and mitigate the hazards that can arise when conducting such simple types of wet lab operations aboard spacecraft. 


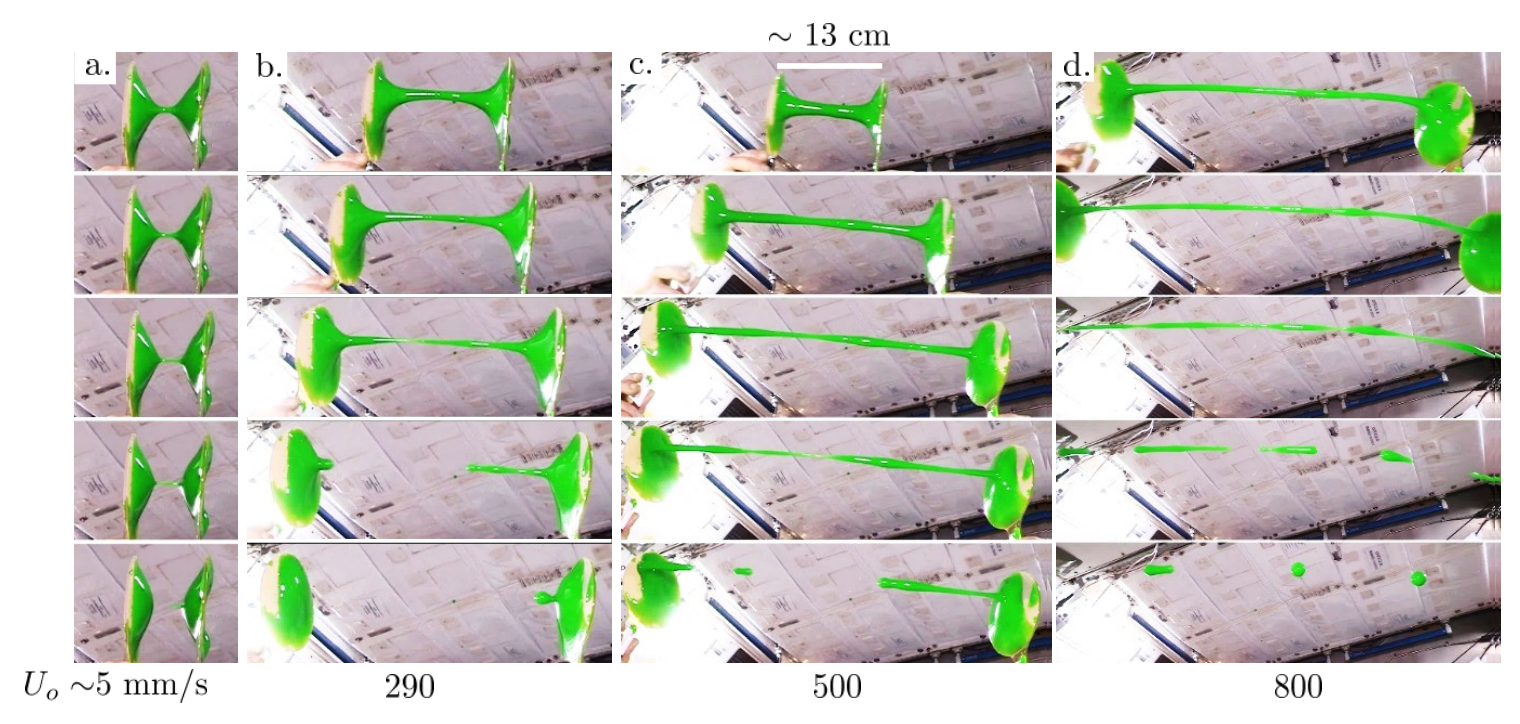

Figure 1.3: Slime in Space demonstration with astronauts observing manually separated viscous liquid bridges formed between $13.34 \mathrm{~cm}$ diameter 'Ping Pong' paddles. a. Paddle separation velocity $U_{o}=0.005 \mathrm{~m} / \mathrm{s}$ is below a critical limit in which rupture occurs at one axial location and no satellite droplets are observed. b. Higher separation velocity $U_{o}=0.29 \mathrm{~m} / \mathrm{s}$ with no satellite droplets observed upon rupture. c. One single $\sim 28 \mathrm{~mm}$ diameter droplet is observed upon bridge rupture, for separation velocity $U_{o}=0.5 \mathrm{~m} / \mathrm{s}$ nearly twice that of b. d. Higher velocity separation $U_{o}=0.8 \mathrm{~m} / \mathrm{s}$ creates unearthly-sized liquid bridge, where rupture occurs at multiple locations along axis and creates multiple visible droplets, $\sim 30 \mathrm{~mm}$ in diameter. 


\section{Chapter 2}

\section{Theory}

The fundamental dynamics of separated liquid bridges are quantified by a single

parameter, the Ohnesorge number $\mathrm{Oh}=\mu /(\rho \sigma R)^{1 / 2}[13]$. The simplicity of a single governing parameter allows for expedious analysis of these computationally intensive non-linear equations in describing the dynamics [14], but the contributions of the axial stretching rate $U_{o}$ are only associated with the boundary condition, which are not represented in Oh. In the present analysis we hope to preserve the intrinsic inertial contributions of liquid bridge instability while addressing the imposed inertia of axial separation, with the motivation to apply them to mitigation techniques for routine pipetting in the open crew quarters of spacecraft. The notation of the problem is identified in Fig. 2.1. In our largely experimental study we seek scale expressions from which to quantify results and establish the bridge rupture regimes for these axially separated liquid bridges. We begin with the momentum equation

$$
\rho \frac{D \boldsymbol{U}}{D t}=\nabla P+\mu \nabla^{2} \boldsymbol{U}+\rho \boldsymbol{g}
$$




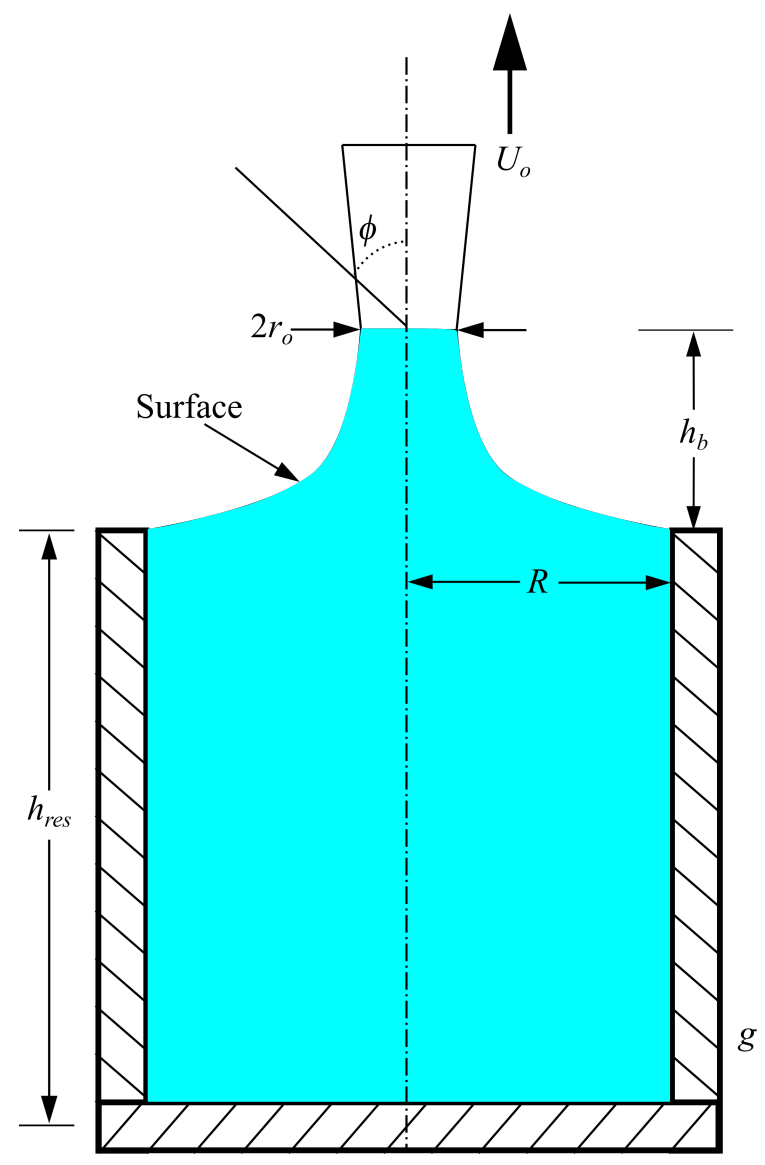

Figure 2.1: Schematic of the pipette liquid-bridge system.

Applying this equation to the pipette withdrawal problem we identify scale quantities such as instrument radius $r_{o}$, local body force $g$, and fluid properties density $\rho$, viscosity $\mu$, surface tension $\sigma$, and contact angle $\theta$. We use these characteristic properties to write the scale version of the axial $z$-component cylindrical coordinate equation

$$
\frac{\rho U_{z}}{t}, \frac{\rho U_{r} U_{z}}{r}, \frac{\rho U_{z}^{2}}{z} \sim \frac{P}{z}, \frac{\mu U_{z}}{r^{2}}, \frac{\mu U_{z}}{z^{2}}, \rho g
$$

In reference to Fig. 2.1 we then choose scales $r \sim r_{o}, z \sim h_{b}$, and $P \sim \sigma / r_{o}$. Additionally mass conservation shows that $U_{r} \sim \gamma U_{z}$ for flows where $\gamma \equiv r_{o} / h_{b}$. At 
present we leave the velocity scale $U_{z} \sim U_{s}$ as unspecified and note that for the liquid bridge geometry to exist at $t=0, h_{b} \sim r_{o}$ such that we choose $z \sim r_{o}$. Additionally, we note that the lifetimes of these liquid bridges, regardless of the inherent fluid properties, is governed by the capillary pressure such that we normalize Eq. 2.2 by $\sigma / r_{o}^{2}$, which yields

$$
\frac{\rho U_{s}^{2} r_{o}}{\sigma}, \frac{\rho U_{s}^{2} r_{o}}{\sigma}, \frac{\rho U_{s}^{2} r_{o}}{\sigma} \sim 1, \frac{\mu U_{s}}{\sigma}, \frac{\mu U_{s}}{\sigma}, \frac{\rho g r_{o}^{2}}{\sigma} .
$$

Examining only the unique terms of Eq. 2.3, we find

$$
\begin{aligned}
\frac{\rho r_{o}}{\sigma} U_{s}^{2} & \sim 1, \frac{\mu}{\sigma} U_{s}, \frac{\rho g r_{o}^{2}}{\sigma} . \\
\mathrm{We}_{s} & \sim 1, \mathrm{Ca}_{s}, \text { Bo }
\end{aligned}
$$

We note that all inertial terms are measured by the Weber number $\mathrm{We}_{s}=\rho r_{o} U_{s}^{2} / \sigma$, with viscous effects and body forces measured by the Capillary number $\mathrm{Ca}_{s}=\mu U_{s} / \sigma$ and Bond number $\mathrm{Bo}=\rho g r_{o}^{2} / \sigma$ respectively. We now assume that the system velocity scale $U_{s}$ is a superposition of the inherent velocity scale $\hat{U}$ of the fluid under axial separation and the imposed boundary velocity $U_{o}$ such that $U_{s}=\hat{U}+U_{o}$. Treating inertial, capillary, viscous, and body forces all as competing terms, and noting the appropriate signs [15] of each term in Eq. 2.4, we temporarily set $U_{s} \sim \hat{U}$ and convert the scale equation of Eq. 2.4 into the algebraic expression

$$
\frac{\rho r_{o}}{\sigma} \hat{U}^{2}+\frac{\mu}{\sigma} \hat{U}-\left(1+\frac{\rho g r_{o}^{2}}{\sigma}\right)=0
$$


Solving for the inherent velocity scale $\hat{U}$ and choosing the real positive root yields

$$
\hat{U}=\frac{\mu}{2 \rho r_{o}}\left((1+4 \mathrm{Su}(1+\mathrm{Bo}))^{1 / 2}-1\right)
$$

This velocity scale characterizes the progression of the bridge towards rupture via the capillary pinch-off mechanism. With the system level velocity scale defined as $U_{s}$ we form $U_{s}=\hat{U}\left(1+U_{o} / \hat{U}\right)$ and substitute into Eq. 2.4 to find

$$
\frac{\rho r_{o} \hat{U}^{2}}{\sigma}\left(1+\frac{U_{o}}{\hat{U}}\right)^{2} \sim 1, \frac{\mu \hat{U}}{\sigma}\left(1+\frac{U_{o}}{\hat{U}}\right), \text { Bo }
$$

Equation 2.8 shows that the magnitude of the LHS is determined by both the inherent fluid parameter $\rho r_{o} \hat{U}^{2} / \sigma$ and the boundary condition velocity ratio $U_{o} / \hat{U}$. To examine the various terms of Eq. 2.8 we employ Eq. 2.7 and look to the boundary condition velocity ratio

$$
\frac{U_{o}}{\hat{U}}=\frac{2 \operatorname{Re}_{o}}{(1+4 \mathrm{Su}(1+\mathrm{Bo}))^{1 / 2}-1} .
$$

The governing parameters contained in $U_{o} / \hat{U}$ are the instrument Reynolds number $\operatorname{Re} \equiv \rho r_{o} U_{o} / \mu \equiv \mathrm{We}_{o} / \mathrm{Ca}_{o}$ and the inherent Suratman number $\mathrm{Su} \equiv 1 / \mathrm{Oh}^{2} \equiv$ $\rho r_{o} \sigma / \mu^{2} \equiv \mathrm{We}_{o}^{1 / 2} / \mathrm{Ca}_{o}$. We note that $\mathrm{Su}$ is the passive capillary Reynolds number with characteristic velocity $\sim \sigma / \mu$. The contributions of input velocity relative to the inherent fluid system response is assessed under two limiting conditions. Assuming inherent inertial-capillary balance or $\mathrm{Su}^{-1 / 2}<<1$ we have

$$
\frac{U_{o}}{\hat{U}} \sim \frac{\mathrm{We}_{o}^{1 / 2}}{(1+\mathrm{Bo})^{1 / 2}}
$$


and Eq. 2.4 becomes

$$
\frac{\rho r_{o} \hat{U}^{2}}{\sigma}\left(1+\frac{\mathrm{We}_{o}^{1 / 2}}{(1+\mathrm{Bo})^{1 / 2}}\right)^{2} \sim 1, \frac{\mu \hat{U}}{\sigma}\left(1+\frac{\mathrm{We}_{o}^{1 / 2}}{(1+\mathrm{Bo})^{1 / 2}}\right), \mathrm{Bo}
$$

and for a visco-capillary balance, $\mathrm{Su}^{1 / 2}<<1$ we have

$$
\frac{U_{o}}{\hat{U}} \sim \frac{\mathrm{Ca}_{o}}{(1+\mathrm{Bo})^{1 / 2}}
$$

with Eq. 2.4 becoming

$$
\frac{\rho r_{o} \hat{U}^{2}}{\sigma}\left(1+\frac{\mathrm{Ca}_{o}}{(1+\mathrm{Bo})^{1 / 2}}\right)^{2} \sim 1, \frac{\mu \hat{U}}{\sigma}\left(1+\frac{\mathrm{Ca}_{o}}{(1+\mathrm{Bo})^{1 / 2}}\right), \text { Bo. }
$$

From both Eq. 2.11 and Eq. 2.13 we note that the only terms preserving both the intrinsic fluid response and the imposed inertia of withdrawal are expansions about $\left(1+\mathrm{We}_{o}^{1 / 2} /(1+\mathrm{Bo})\right)$ and $\left(1+\mathrm{Ca}_{o} /(1+\mathrm{Bo})\right)$ respectively, and when body forces are negligible $\mathrm{Bo} \ll 1$ they reduce to $\left(1+\mathrm{We}_{o}^{1 / 2}\right)$ and $\left(1+\mathrm{Ca}_{o}\right)$. It is the limiting cases of $U_{o} / \hat{U}$ that guide our choices of dimensionless parameters, $\mathrm{We}_{o}^{1 / 2}$ and $\mathrm{Ca}_{o}$, to construct regime maps of these events. The ratio of $U_{o} / \hat{U}$ allows for the input contributions of instrument geometry and withdrawal rate to remain $O(1)$ with a single term and examine the relative effects of inherent fluid properties and separation velocity. Noting the relationship between the Ohnesorge and Suratman numbers we are able to differentiate between the dominate forces inherent to the fluid and account for effects of the fluid properties when these liquid bridges become elongated and rupture. In the following chapters we present regime maps organized by $\mathrm{We}_{o}^{1 / 2}$ and $\mathrm{Ca}_{o}$. The full non-dimensional set of equations governing these types of flows 
is presented in Appendix B from which it is confirmed that only two parameters, $\mathrm{We}_{o}^{1 / 2}$ and $\mathrm{Ca}_{o}$, determine the system response regardless of limiting cases. 


\section{Chapter 3}

\section{Experiments}

All drop tower experiments are conducted using the Dryden Drop Tower [16] located at Portland State University pictured schematically in Fig. 3.1. The $22.2 \mathrm{~m}$ tall tower provides approximately $2.1 \mathrm{~s}$ of free fall where the experiment rig experiences a near weightless state with maximum local accelerations $\lesssim 10^{-4} g_{o}$. The experiment rig is depicted in Fig. 3.2. Linear actuators and spring-mass systems are used to achieve instrument withdrawal rates of $0.02 \leq U_{o} \leq 1.2 \mathrm{~m} / \mathrm{s}$. A Panasonic WX970 HD camcorder and Phantom v4.3 high speed camera are employed with frame rates ranging from 120 to 7300 fps, respectively. Pixel densities from 128x512 px up to $3840 \times 2160$ (4k) HD allowed for images across experiments that maintain an approximate scale factor of $100 \mu \mathrm{m} / \mathrm{px}$. The experiments are back-lit by diffused LED panels. On-board DC power sources supply power to all image and experiment equipment. An ADXL-345 accelerometer with an Arduino controller activates instrument withdrawal sequences upon the near step-reduction of gravity. When local accelerations decrease sufficiently below $g_{o}$ the instrument tip initially plunged into to the test fluid is withdrawn creating, elongating, and rupturing the liquid bridge. Multiple common scientific cannula geometries are employed as shown in Fig. 3.3 with transparent 3D printed SLA reservoirs of varying inner diameters. 
The test fluids are distilled water and water-glycerol mixtures of varying viscosities and surface tensions listed in Table 3.1.

a.

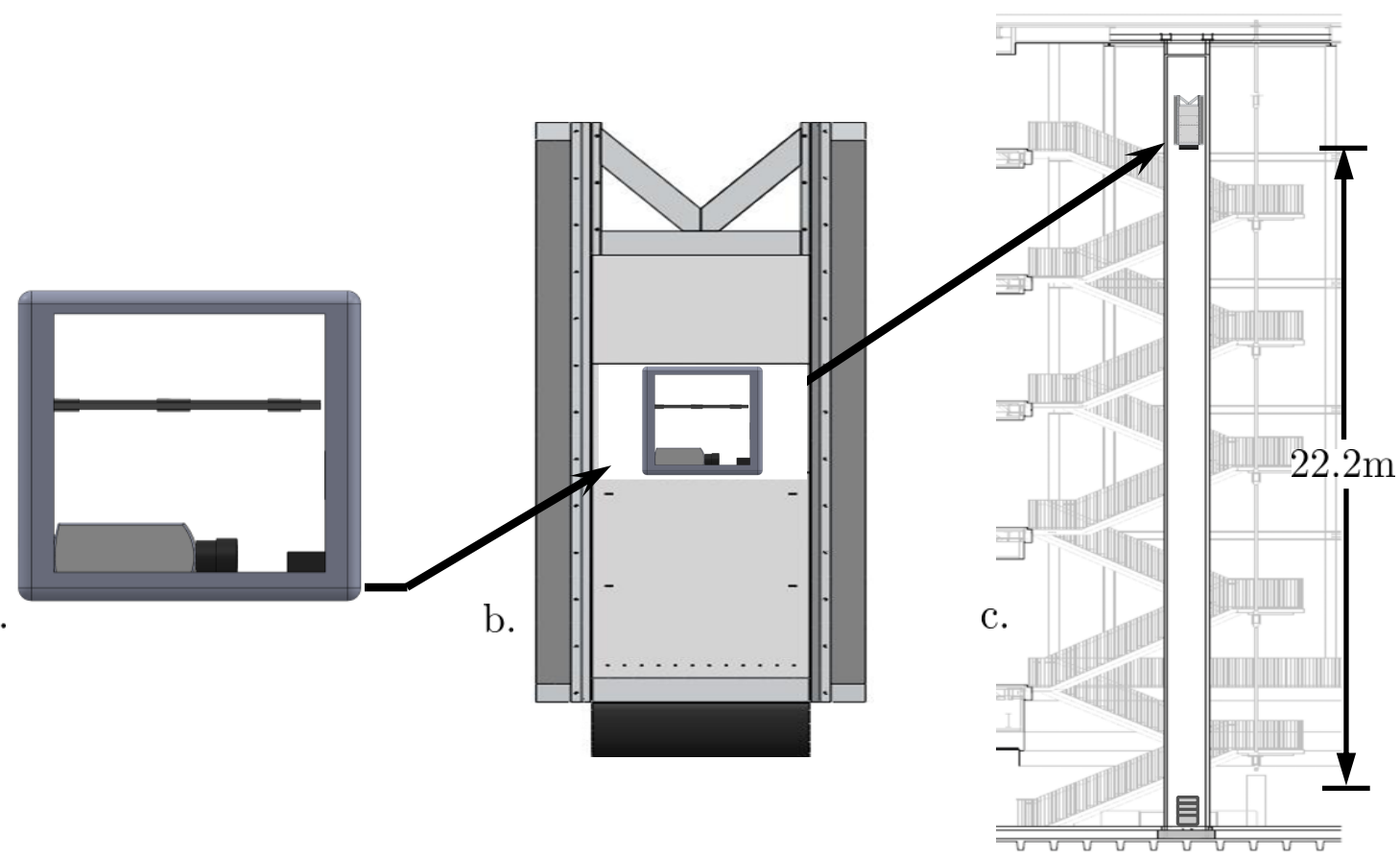

Figure 3.1: Dryden drop tower and experiment rig schematic: a. experiment rig with support equipment b. drag shield c. tower and elevation schematic. 


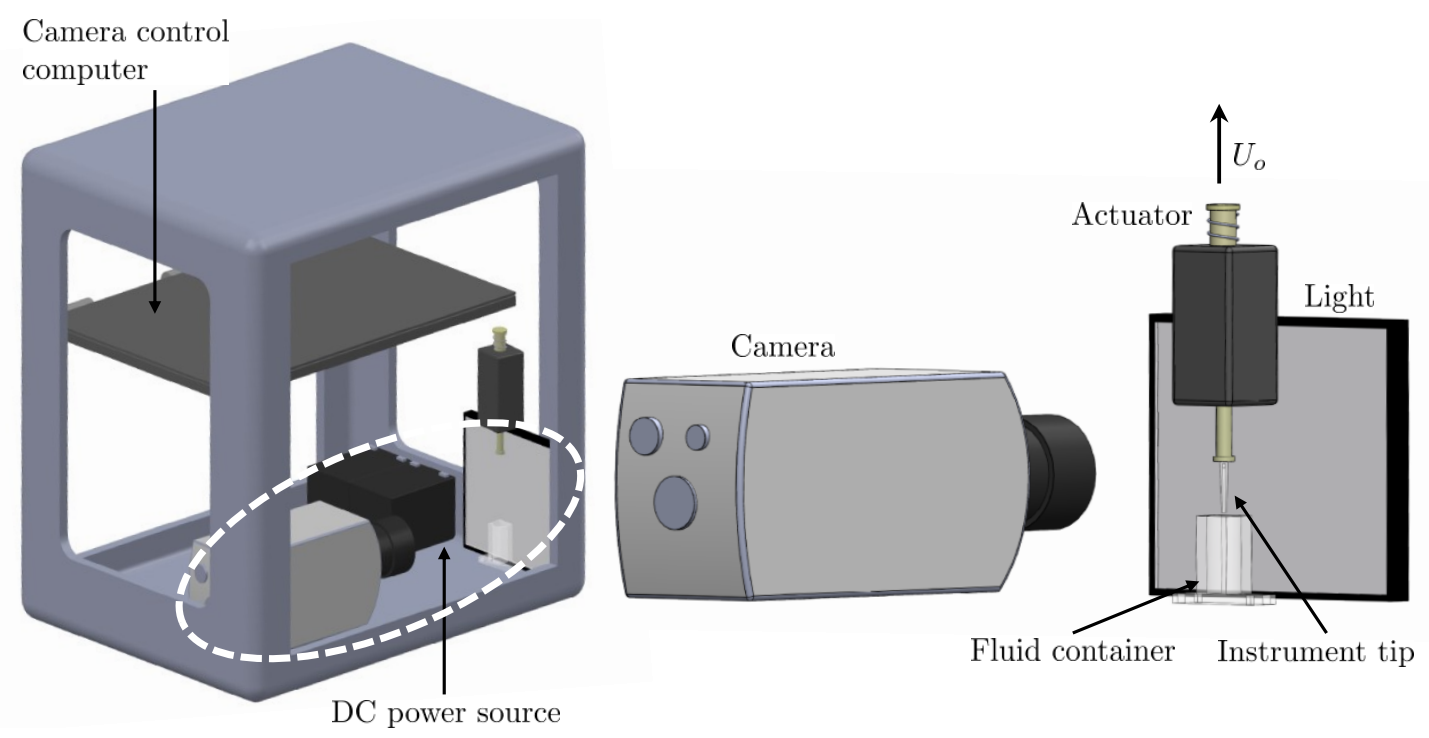

Figure 3.2: CAD schematic of experiment rig assembly. Breakout view of the orientation and nomenclature for the imaging, lighting, and fluid withdrawal systems. Not shown are the Arduino controller circuit and assorted camera/power cables.

a.

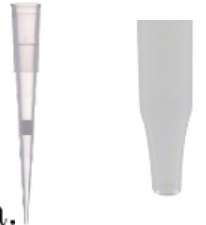

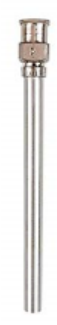

$\mathrm{b}$

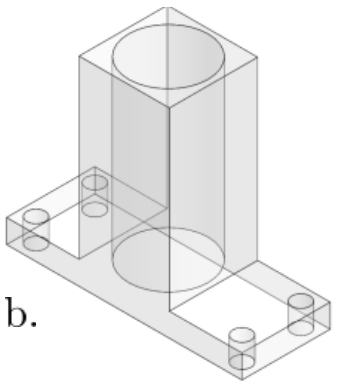

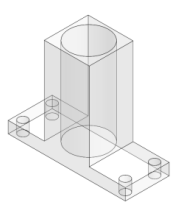

Figure 3.3: a. Cannula geometries employed with $r_{o}=0.5,1.8$, and $2 \mathrm{~mm}$ respectively $\mathrm{b}$. Isometric CAD view of container geometries employed $R=10$, 5, 3, 2, $1 \mathrm{~mm}$ respectively. All containers maintained the same aspect ratio $h_{\text {res }} / R=3$.

A supplementary data set of terrestrial experiments is also conducted using the same drop rig apparatus. The terrestrial experiments are performed on a Newport RS4000 vibration isolation table. Following each experiment, all video data is con- 
verted to still image files and analyzed using open-source image analysis software FIJI [17]. Withdrawal rate, bridge length, bridge diameter, and satellite droplet numbers, dimensions, and velocity trajectory data are tabulated. Over 90 drop tower tests are conducted in support of pipette droplet generation and mitigation application demonstrations alone.

Table 3.1: Fluid properties at room temperature

\begin{tabular}{|c|c|c|c|}
\hline $\begin{array}{c}\text { Vol. Fraction } \\
\left(\mathrm{H}_{2} \mathrm{O} / \mathrm{Gly}\right)\end{array}$ & $\mu(\mathrm{kg} /(\mathrm{m} \cdot \mathrm{s}))$ & $\rho\left(\mathrm{kg} / \mathrm{m}^{3}\right)$ & $\sigma(\mathrm{mN} / \mathrm{m})$ \\
\hline \hline $1 / 0$ & 0.00089 & 1000 & $69.11 \pm 0.21$ \\
\hline $44.8 / 55.2$ & 0.01 & 1154 & $62.79 \pm 0.1$ \\
\hline $20 / 80$ & 0.08 & 1216 & $66.52 \pm 0.03$ \\
\hline $3 / 97$ & 0.68 & 1253.8 & $64.2 \pm 0.005$ \\
\hline
\end{tabular}




\section{Chapter 4}

\section{Numerical Analysis}

The complexities of solving the non-linear systems of equations that describe these types of free surface flows are beyond the scope of this work. We are primarily concerned with the first order production of ejected droplets upon rupture and the limiting stable configurations of these liquid bridges when instruments become withdrawn from a fluid container. The data serves as benchmarks for numerical analyses also pursued herein using Surface Evolver and Volume of Fluid CFD software to asses the contributions of system inputs (i.e. withdrawal rates, instrument geometry, and fluid properties) to the outputs of liquid bridge dimensions and ejected droplet properties.

\subsection{Liquid Bridge Statics for Pipetting Systems}

Numerical computations are performed using the Surface Evolver Fluid Interface Tool, SE-FIT, [18] software to determine or confirm static liquid bridge interface configurations and their stability for a variety of geometries and fluid properties. SE-FIT is an open source free surface solver using K. Brakke's Surface Evolver algorithm [19] as a computational engine within a customizable GUI, referred to as the Fluid Interface Tool. In this case the SE-FIT software is efficiently employed using 
the 'Pipette' pre-built model. The Pipette pre-built model defines system geometry, constraints, and boundary conditions. A schematic of the model was provided in Fig. 2.1 noting pipette outer radius $r_{o}$, pipette retraction angle $\phi$, reservoir radius $R$, height $h_{\text {res }}$, liquid bridge height above the container rim $h_{b}$, and contact angle $\theta$. The gravitational acceleration $g$, density $\rho$, and surface tension $\sigma$ are collected in the unit Bond number, $\mathrm{Bo}_{u n i t}=\rho g / \sigma$.

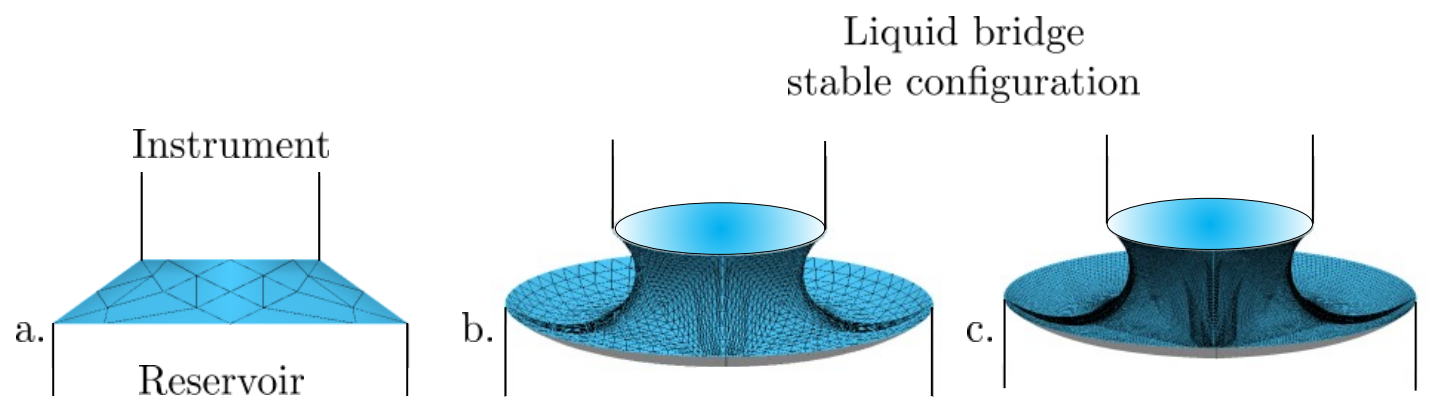

Figure 4.1: Numerically computed free surface profiles for the pipette prebuilt model. a. initial condition, b. rough convergence, and c. deeply refined converged solution

Gravity conditions are controlled by the $\mathrm{Bo}_{\text {unit }}$ value. The pipette pre-built model constrains the liquid to the pipette tip outer radius and reservoir inner lip using a pinned boundary condition. The initial condition of the model is shown in Fig. 4.1a, where a truncated conical surface mesh pinned at the pipette tip and reservoir edge defines the free surface. The pipette model fluid volume of the liquid bridge is set to zero. From a practical standpoint this condition is observed during pipetting events. Prior to pipetting the reservoir interface is initially flat, then responds to an instrument being withdrawn. There is no volume of fluid in these liquid bridges, only an interfacial response to an instrument being withdrawn further and further from the initial interface until rupture occurs. Examples of refinements 
of the mesh during the simulations are presented in Fig. 4.1b. and c.

The SE-FIT numerical analysis of the limiting stable bridge heights provides a prediction of liquid bridge rupture heights for extremely low velocity pipette removal. Both instrument Weber number $\mathrm{We}_{o}=\rho r_{o} U_{o}^{2} / \sigma$ and Capillary number $\mathrm{Ca}_{o}=\mu U_{o} / \sigma$ contain the dynamic system property of withdrawal velocity $U_{o}$ such that, when comparing to the SE-FIT results, requires $\mathrm{We}_{o} \ll 1$ and $\mathrm{Ca}_{o} \ll 1$. All numerical cases explored herein employ $\phi=0$. The dimensionless stable bridge height limits are discussed in connection with Fig. 5.6 for a variety of $\mathrm{Bo}_{\text {unit }}$.

\subsection{Liquid Bridge Dynamics For Pipetting Systems}

To analyze the dynamics of liquid bridge rupture following instrument withdrawals from liquid reservoirs, CFD numerical simulations are also pursued using the opensource code Gerris [20,21]. While it is accepted that using CFD packages as a means to predict satellite droplet generation is solely mesh dependent [22], the Gerris software is employed herein to asses the accuracy of $O(1)$ ejected droplet sizes and regime map transitions for the instrument withdrawal events. All simulations are run using the Cartesian grid-based Finite Volume of Fluid (VOF) method with Adaptive Mesh Refinement (AMR). Figure 4.2 shows for the 2-D axisymmetric model a select bridge rupture event for water and $1 \mathrm{~mm}$ outer diameter instrument with $U_{o}=0.5$ $\mathrm{m} / \mathrm{s}$.

To simplify the implementation of moving boundary conditions a relative motion approach was taken whereby, rather than withdraw the instrument from the liquid, the fluid is drained from the bottom of the reservoir. The reservoir walls are assigned a slip condition and prescribed contact angle $\left(\theta=90^{\circ}\right)$ to reduce the 
influence of viscous dissipation from the moving walls. The simulation solves the dimensionless Navier-Stokes and phase transport equations with dimensionless groups and fluid parameters defined in a user specified simulation file allowing for direct analogy to the physical system. For the case of the pipette instrument and reservoir geometry model, the drain velocity, viscosities, densities, and contact angle are defined explicitly for both fluid phases. Ratios of inertial-viscous forces are defined by the Reynolds number $\left(\operatorname{Re}=\rho r U_{o} / \mu\right)$ with inertial-capillary forces defined by the Weber number (We $\left.=\rho r U_{o}^{2} / \sigma\right)$. Gravity effects are captured by the Froude number Fr $=U_{o} /\left(g r_{o}\right)^{1 / 2}$. Run time steps are calculated via the Courant-FriedrichsLewy (CFL) condition and shortest capillary wave in the numerical analysis with the default $\mathrm{CFL}=0.8$ being used.

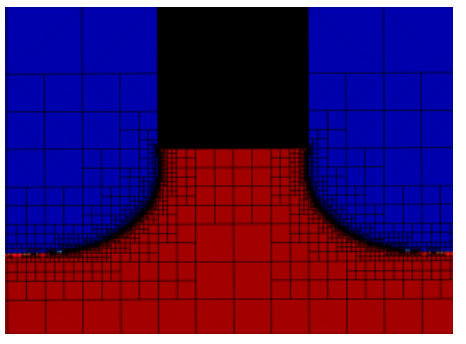

$t_{o}=0 \mathrm{~s}$

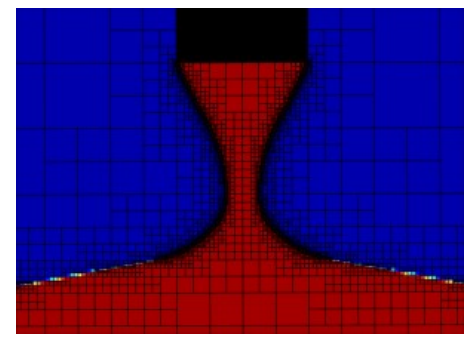

0.0009

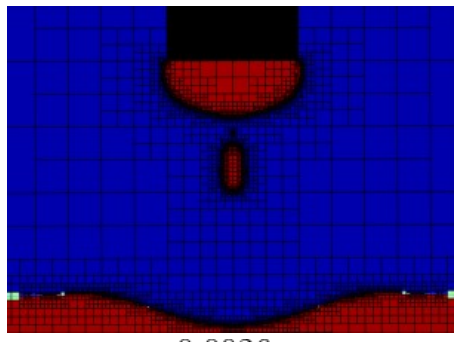

0.0026

Figure 4.2: Example dynamic meshing in Gerris using the AMR algorithm highlighting increased mesh density at areas with large curvature. The time sequence highlights meshing of the interface at $t=0 \mathrm{~s}$, during liquid bridge necking, and when the bridge ruptures and a droplet is ejected. Dimensionless parameters defining this simulation are $\mathrm{We}_{o}^{1 / 2}=0.37$ and $\mathrm{Ca}_{o}=0.0012$.

The proprietary software STAR-CCM+ was also employed for further numerical analysis and to asses higher order effects of pipette liquid bridge rupture when fluid is entrained within the instrument. Additional STAR-CCM+ models include a super-hydrophobic instrument outer surface simulation for potential mitigation 
applications. A similar 2-D, rotationally symmetric, relative motion VOF model was built for simplicity. However, due to the more complex nature of the equations solved by STAR the computational time (approximately 20 hours/simulation) only allowed for small number of test cases (6) to be completed. 


\section{Chapter 5}

\section{Results}

\subsection{Experiments}

Even a brief review of the literature suggests that liquid bridge rupture leads to a seemingly endless cascade of ruptures which in turn leads to an equal number of satellite droplet ejections with decreasing droplet sizes [5]. At some point fixed resolution imaging will fail to detect the minuscule, high speed droplets $U_{\text {drop }} \sim\left(\sigma /\left(\rho r_{\text {min }}\right)\right)^{1 / 2}$, where $r_{\text {min }}$ is defined as the liquid bridge radius just before pinch off. We define this limit as the 'no observable satellite droplet' limit. For any imaging system with a defined focal plane there is a fixed pixel density that defines the length scale of a single pixel. If an ejected droplets size is less than the length scale of a single pixel in the focal plane, it obviously cannot be resolved and observed. The same is true for the speeds of these ejected droplets. For a given ejected droplet, if the ejection velocity is greater than the length scale of a pixel over the exposure time of the camera then the droplet will appear as a faint blur. For the high speed imaging system employed, the exposure time is 40 $\mu \mathrm{s}$ which corresponds to an ejection velocity of $\sim 2.5 \mathrm{~m} / \mathrm{s}$ in order for the droplet to move at least one pixel prior to a single frame imaged in the video sequence. 
For droplets traveling at such speeds upon ejection the corresponding scale radii of said droplet must be $\sim 10 \mu \mathrm{m}, 10$ times less than the size limit of our observation $(\sim 100 \mu \mathrm{m})$. While there does exist a velocity limit required to resolve these ejected droplets, the 'no observed' limit reported herein is most limited by the size of these droplets. However, for practical reasons we are mostly interested in the largest droplets produced during such ruptures. Our experiments produce ejected droplets in the range of $100 \mu \mathrm{m} \lesssim r_{\text {drop }} \lesssim 4 \mathrm{~mm}$, with which we categorize observed droplet ejections based on the dimensionless parameters highlighted in Chapter 2. From our experiments, 'satellite droplets' produced are on the order of $\delta<0.2$, 'mother droplets' are on the order of $0.2 \leq \delta<1$, and 'ligament' ejections are on the order of $\delta \geq 1$, where $\delta \equiv r_{d r o p} / r_{o}$. Ligament ejections typically produce a droplet dispersion as observed by Marrmotant [7]. We report an equivalent droplet radius as determined using $r_{\text {equiv }}=\left(\sum_{i}^{n} r_{i}^{3}\right)^{1 / 3}$ where droplet radii $r_{i}$ are measured from the video footage. Fig. 5.1 provides sample images of the ejection types. From the two parameter scaling approach pursued in Chapter 2 we construct regime maps highlighting when such droplet ejections arise based on the input parameters of $\mathrm{We}_{o}^{1 / 2}$ and $\mathrm{Ca}_{o}$. From Fig. 5.2 it is clear that for sufficiently low values of $\mathrm{We}_{o}^{1 / 2}$ and $\mathrm{Ca}_{o}$ the ejected droplet sizes are typically minimal, $\delta<0.2$. As the withdrawal inertia increases ejected droplet sizes increase as well. Experimental observations show that critical values of transitions between ejected droplet sizes occur at approximately $\mathrm{We}_{o}^{1 / 2} \sim 0.7$ for satellite-mother droplets and $\mathrm{We}_{o}^{1 / 2} \sim 5$ for mother-ligament ejection transitions within the inertial-capillary balance. For the inertial-visco-capillary regime we observe $\mathrm{We}_{o}^{1 / 2} \sim 0.2$ for the satellite-to-mother droplet transition, and $\mathrm{We}_{o}^{1 / 2} \sim 1$ for mother-to-ligament transition. A noteworthy distinction between 1-g 
and low- $g$ data is that ligament ejections with ejected droplets of radii $r_{\text {equiv }} \geq r_{o}$ appear only to occur in low-gravity environments. This is due in part to the lack of draining that would otherwise cause these liquid bridges to continually thin to the point of rupture, decreasing the total ejected volume of fluid.

The terrestrial work of Zhuang et al. [23] characterizes similar types of stretched liquid bridges up to rupture using a single parameter $\mathrm{Oh}=\mu /(\rho r \sigma)^{1 / 2}$ as has been observed previously. As the stretching velocity increases for constant Oh, so does the critical bridge height at rupture. Similarly, as Oh increases we see an increase in bridge height for constant withdrawal rate owing to the increase in viscous resistance, slowing the speed at which inherent capillary forces cause these bridges to break as highlighted in Fig. 5.3. One would expect that an increase in viscosity for constant withdrawal speeds would allow for larger droplet volumes to be produced at breakup, although from the experimental ranges of $U_{o}$ and $\mu$ employed in our experiments this increase is negligible when the previous definitions of ejected droplet size are applied. Typical droplet sizes for the viscous fluids employed are roughly $0.5<\delta \leq 1$. The results obtained experimentally remain within the 'mother' droplet definition with very few viscous bridge ruptures producing ligaments, yet we note an increase in $r_{\text {drop }}$ due to this increased viscous resistance for the inertial-visco-capillary region. 

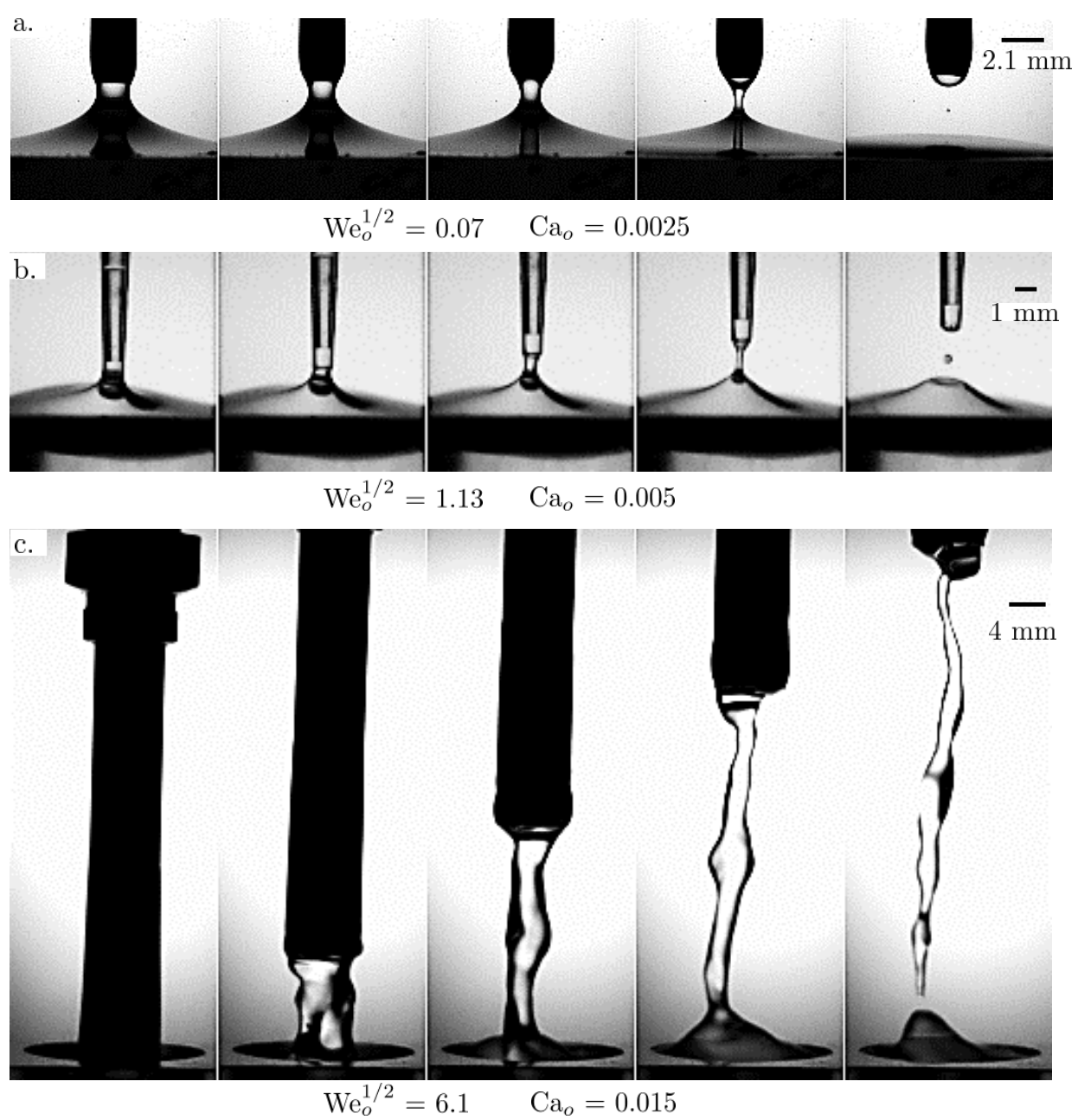

Figure 5.1: Three Distinct liquid ejection regimes observed during low- $g$ experiments. a. 'Satellite' droplet, $\delta=0.06$ (image sequence at $105 \mathrm{~Hz}$ ) b. 'Mother' droplet, $\delta=0.6$ (image sequence at $770 \mathrm{~Hz}$ ) and c. 'Ligament ejection', $\delta=1.05$ (image sequence at $150 \mathrm{~Hz}$ ) 

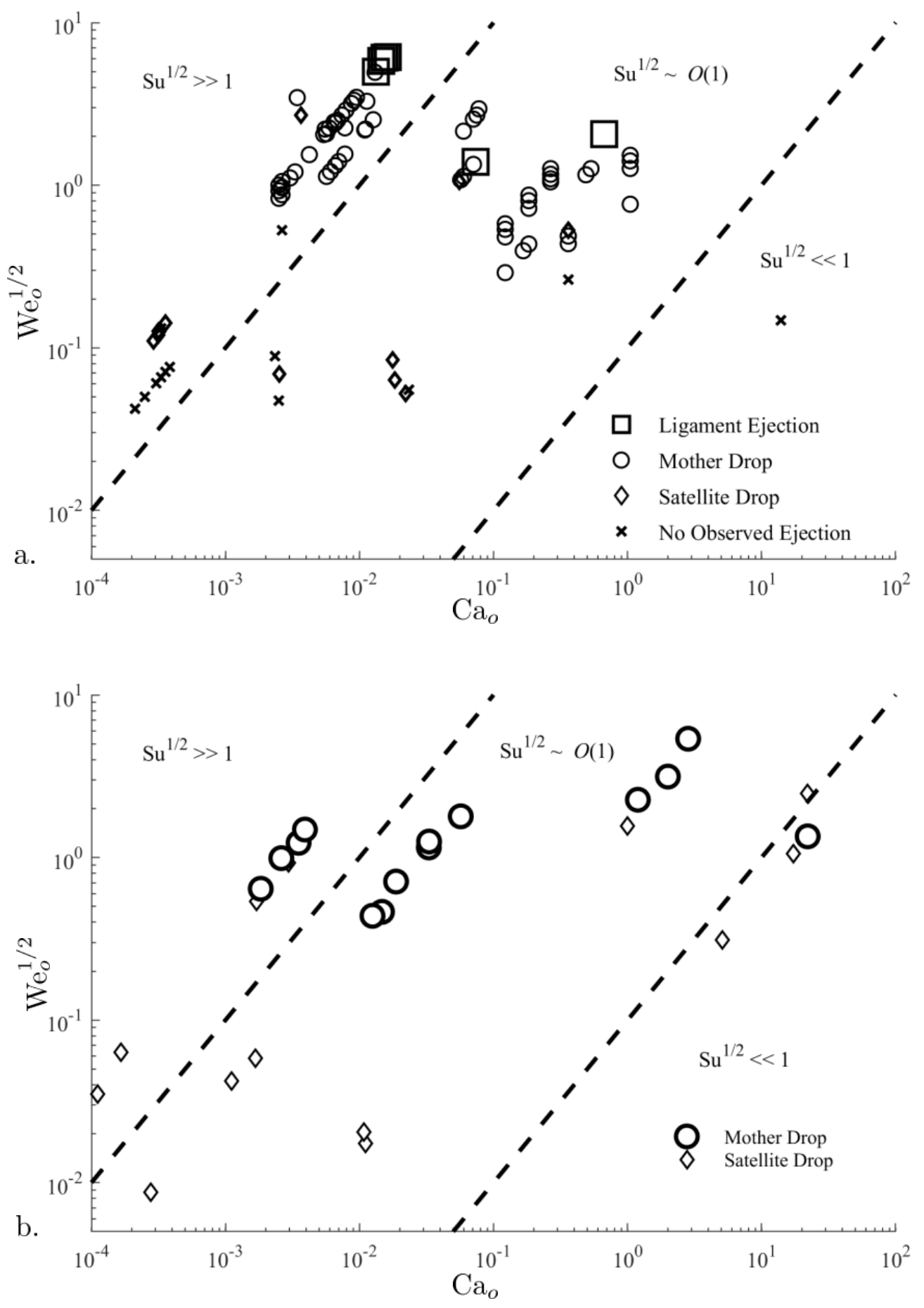

Figure 5.2: Regime maps for droplet ejections in a. low- $g$ and b. $1-g_{o}$. Large volume ligament ejections appear to be exclusive to low- $g$ environments where $g$ induced drain does not act to thin these bridges while elongation occurs. 


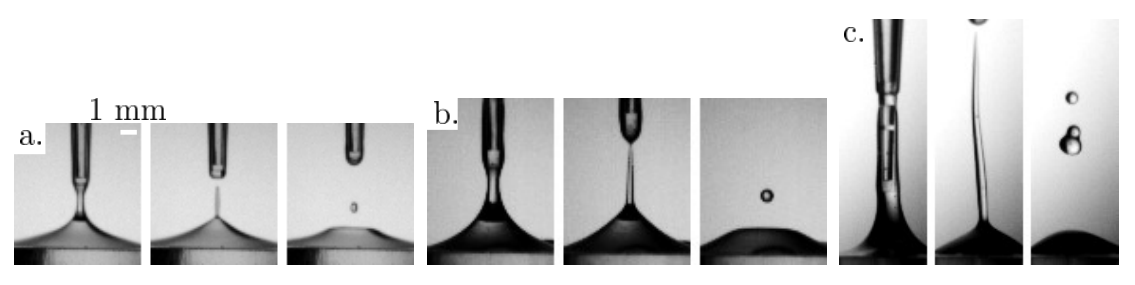

Figure 5.3: Approximately constant $\mathrm{We}_{o}^{1 / 2} \sim 1.3$ withdrawals for $1 \mathrm{~mm}$ OD instrument (scale factor is constant for all images) with increasing $\mathrm{Ca}_{o}$ via increasing viscosity a. $\mathrm{Ca}_{o} \sim 0.006 \mathrm{~b} . \mathrm{Ca}_{o} \sim 0.007$ and c. $\mathrm{Ca}_{o} \sim 0.07$. Image sequence highlights critical dynamic bridge heights and ejected droplet sizes increase with viscosity.

Recalling the limits of Eq. 2.9 we find the dimensionless groups that define these type of droplet ejection events. From the fluid properties, geometries, and withdrawal velocities achieved in experiments we find that the majority of the data in the droplet ejection regime map of Fig. 5.2 is within the inherent inertial-capillary limit and inertial-visco-capillary regime for both $\mathrm{Su}^{1 / 2} \gg 1$ and $\mathrm{Su}^{1 / 2} \sim O(1)$, respectively. The high viscosities necessary to examine the visco-capillary regime could not be tested in drop tower experiments due to the excessive liquid bridge lengths $(\gtrsim 50 \mathrm{~mm})$ at rupture and short duration of low- $g$ time provided by the DDT. However, from the informal 'Slime in Space' demonstration data we are able to estimate relative bridge retraction speeds and the resulting dimensions of ejected droplets produced from an extremely viscous fluid $(\mu \sim 200 \mathrm{~kg} /(\mathrm{m} \cdot \mathrm{s}))$. Shown in Fig. 5.5 is the droplet ejection regime map with the 'Slime in Space' data included. From the small sample size of tests satisfying the $\mathrm{Su}^{1 / 2}<<1$ condition, we observe a similar trend in droplet ejection transitions with an inertial shift to higher retraction velocities required to produce similar ejection types. The 'Slime in Space' demonstrations show that for such viscous fluids to produce observable droplets 
upon rupture the withdrawal parameter $\mathrm{We}_{o}^{1 / 2}$ must be $\sim 10$, a remarkable increase in the input inertia required when working with highly viscous fluids.

From the experimental data, preliminary boundaries for ejection types are approximated through the ranges of $\mathrm{Su}^{1 / 2}$, which define regimes based on the inherent forces acting on these fluids. Shown in Fig. 5.4 are the approximated regime transition borders based on the input forces defined by $\mathrm{We}_{o}^{1 / 2}$ and $\mathrm{Ca}_{o}$, defining when one could expect either satellite, mother, or ligament sized ejected droplets. While the transitions of droplet ejection regimes between large and $O(1)$ values of $\mathrm{Su}^{1 / 2}$ are nearly similar, the extreme increase in input inertia relative to capillary forces necessary to produce similar droplet ejection types for highly viscous fluids is remarkable. 


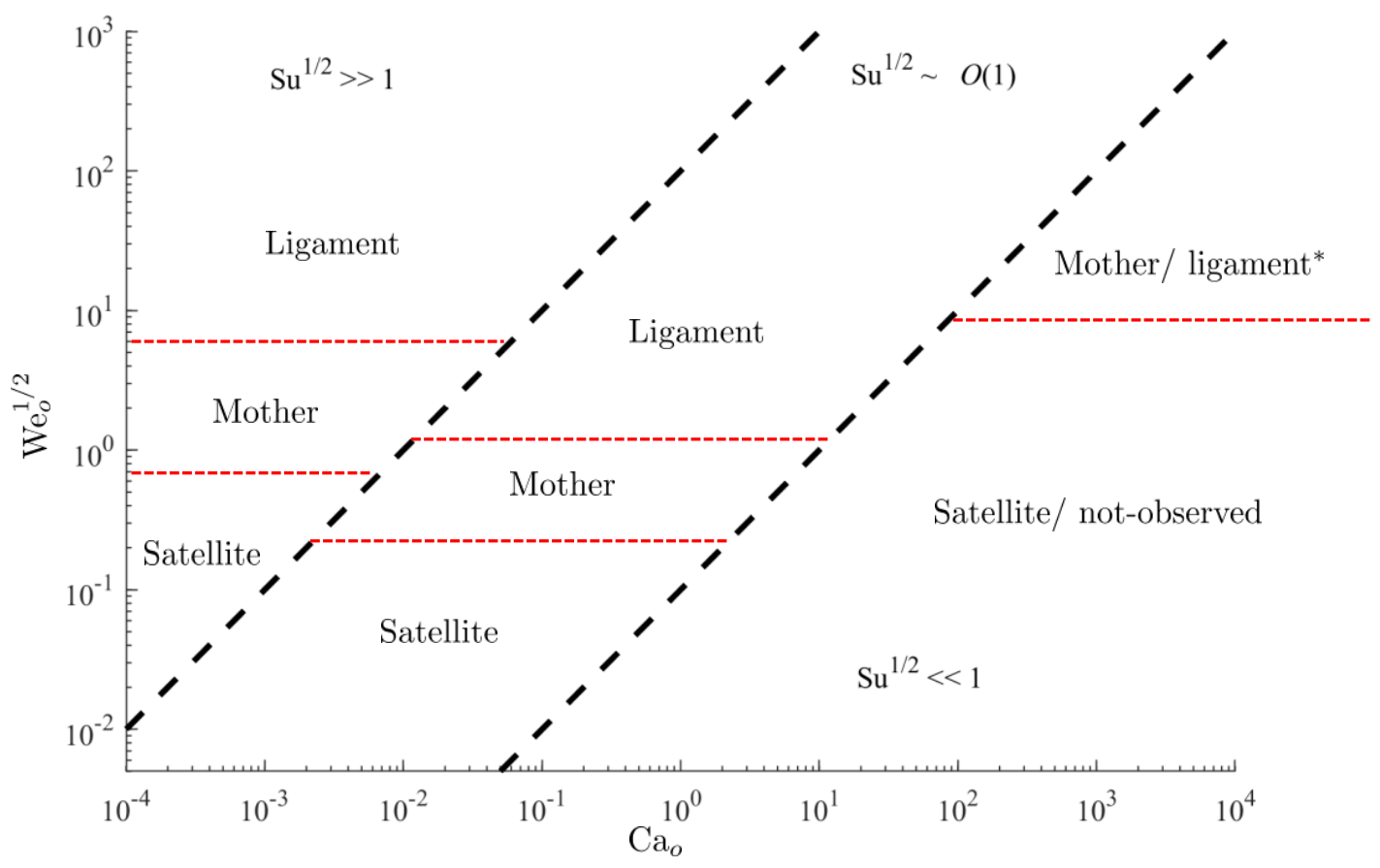

Figure 5.4: Approximate regime boundary transitions in low- $g$ between satellite to mother droplet ejections and mother-ligament droplet ejections as observed experimentally for the three distinct fluid regimes determined by $\mathrm{Su}^{1 / 2}$. Note that ligament* is an assumed ligament regime. Equivalent droplet radii from the Slime in Space demonstration used to calculate corresponding $\delta$ 's are small due to the large boundary geometry from the ping pong paddles used to separate these liquid bridges. 

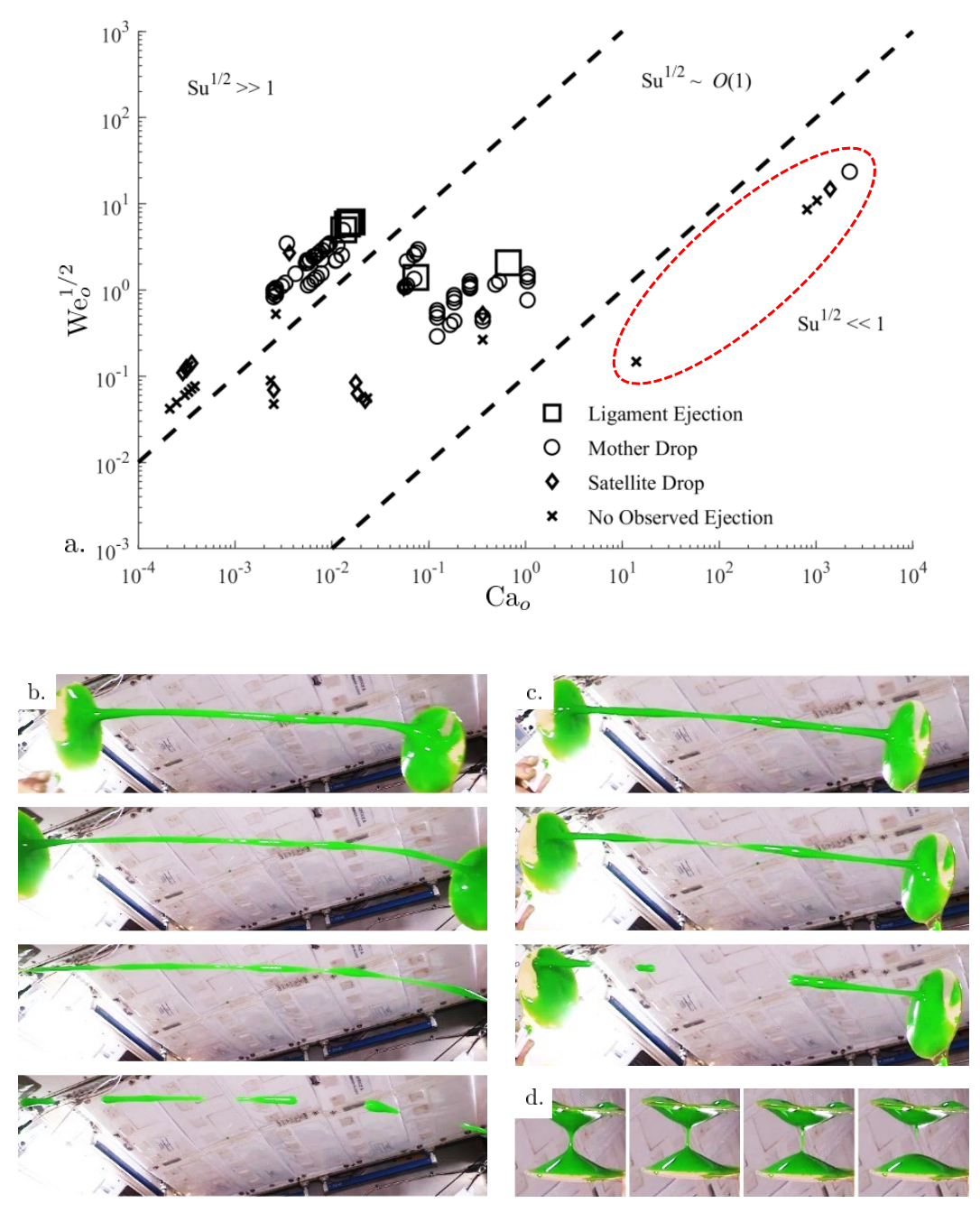

Figure 5.5: a. Regime map of Fig. 5.2 with the 'Slime in Space' demonstration experiments included. b. Unearthly sized liquid ligament stretched beyond stable limits in low- $g$ leading to breakup producing multiple droplets. c. Slower retraction breakup with Slime where only a single droplet is produced with ligaments returning back to the bulk fluid on paddles before further rupture can occur. d. Slow quasi-steady Slime bridge rupture where no satellite droplets are observed. 


\subsubsection{Uncertainty Analysis}

The modest levels of precision of the linear stages employed for the instrument retractions leads to certain variations in imposed vertical motions which in turn lends itself to uncertainties in the reproducibility of experiments. Image processing uncertainties add to the overall accuracy estimations for the data collected. When viewed by the high speed camera, small lateral perturbations of the retracting instruments are observed and quantified $(\approx \pm 40 \mu \mathrm{m})$. Such perturbations lead to premature instability or even prolonged stability of the elongating liquid bridges. However, by comparisons to the 2-D numerical rotationally symmetric computations, ejected droplet volumes due to off-axis experimental controls appear to be negligible.

On the other hand, image analysis errors stemming from resolutions, exposure time, and frame rate limitations lead to certain quantifiable uncertainties. Though multiple cameras are used, the length scale of each pixel for a given camera was fixed at roughly $100 \mu \mathrm{m} / \mathrm{px}$. Errors for larger ejected droplet volumes are minimal as the pixel length relative to the droplet dimension is less than approximatley $2 \%$. However, for the smaller satellite droplets produced, reported radii contain considerable uncertainty as the measured lengths of these satellite droplets approach the pixel length scale. Droplet dimension errors for these small satellite droplets produced from the smallest canulla geometries employed are nearly 50\%. Though the errors produced from these small scale droplets is considerable, we report the ejected droplet dimensions liberally as the reported dimensions are an approximate maximum possible size of said droplets. 'No observed ejection' observations reported are not guaranteed as no ejection events for the corresponding parameters. If a droplet is ejected in such a case the droplet diameter is at most $<100 \mu \mathrm{m}$. Droplet 
volumes of this size are $\lesssim 1 p \mathrm{~L}$, which may or may not be of consequence. As an example, for a low- $g$ evaporation rate of $220 \mathrm{~g} /\left(\mathrm{m}^{2} \mathrm{hr}\right)$ at $22^{\circ} \mathrm{C}$ in a $40 \%$ relative humidity environment it would take 15 minutes for the largest of these undetectable water droplets to evaporate [24].

\section{$5.2 \quad$ Numerics}

\subsubsection{Statics}

The 3-D static SE-FIT Pipette pre-built numerical model discussed in Chapter 4 allows for a parameter sweep through a wide variety of liquid bridge boundary conditions, $g$ levels, and $g$-level orientations acting on the fluid. Shown in Fig. 5.6 are dimensionless critically stable pipette bridge configurations with select experimental low velocity quasi-steady retraction critical bridge heights provided for comparison. As one would expect, as $\mathrm{Bo}_{\text {unit }}$ is increased we observe a decrease in stable heights as a function of $r_{o} / R$. An increase in $r_{o} / R$ for constant $\mathrm{Bo}_{\text {unit }}$ shows a decrease in stable heights as well. Due to the imposed zero volume bridge constraint, as $r_{o} / R$ approaches 1 we would expect a decrease in stable liquid bridge heights to minimize surface energy of the interface. We also observe an interesting transition from axisymmetric to asymmetric free surface configurations. For all $\mathrm{Bo}_{\text {unit }}$ we compute transition between symmetric to asymmetric states when $r_{o} / R>0.5$. The stable asymmetric configurations coupled with liquid bridge rupture dynamics and subsequent satellite droplet production introduce additional off-axis complexities into possible predictions on the size, speed, and trajectories of ejected droplets. 


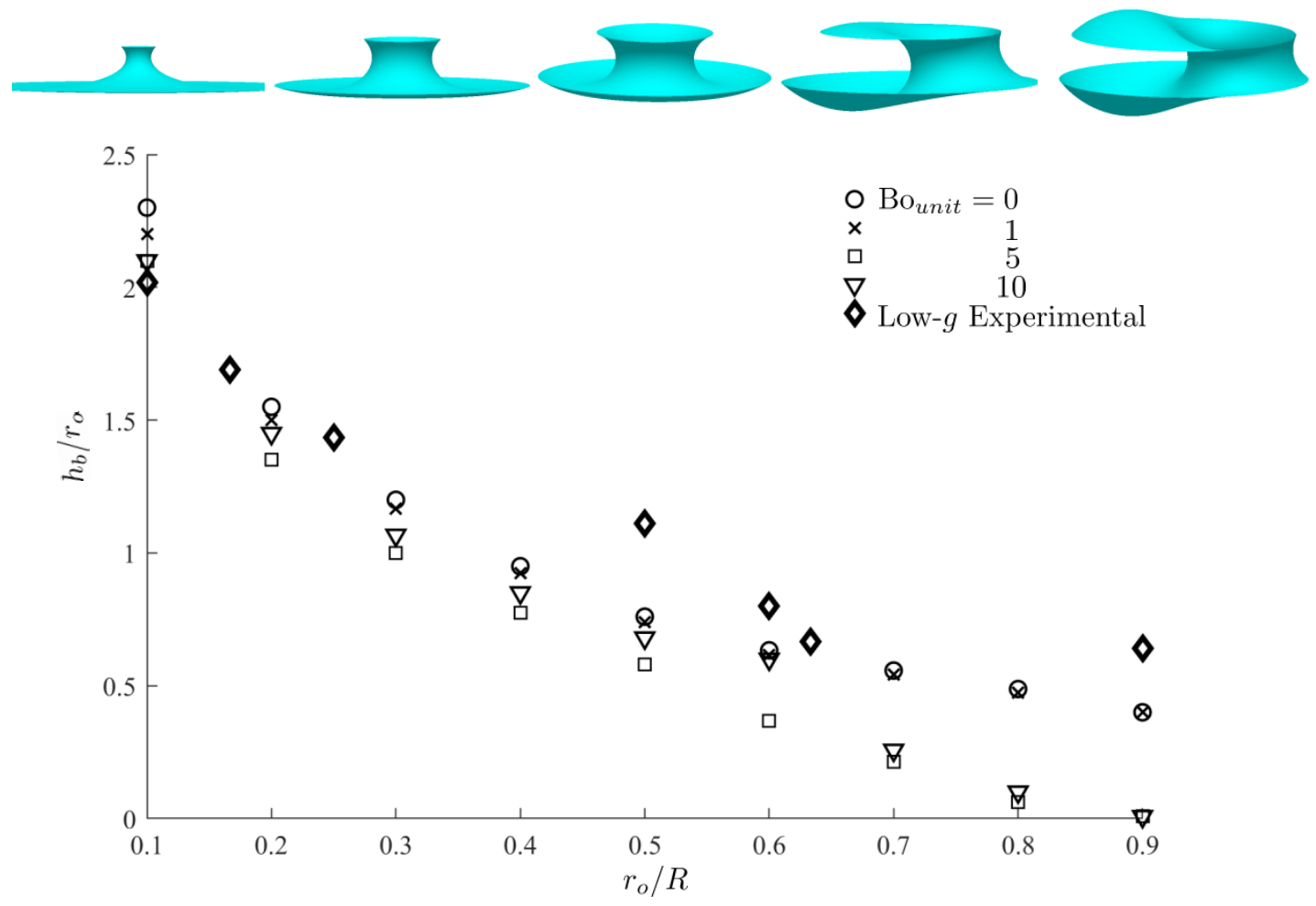

Figure 5.6: Marginal stability curves computed by SE-FIT for a range of $\mathrm{Bo}_{\text {unit }}$. Quasi-steady experimental bridge heights are included for comparison. Selected images of the liquid bridge marginal stable configurations are included above plot to highlight asymmetric configurations for all $\mathrm{Bo}_{\text {unit }}$ when $r_{o} / R>0.5$.

\subsubsection{Dynamics}

The Gerris and STAR-CCM+ numerical simulations are bench-marked by experimental results. Although the models are simplifications, the Gerris model in particular provides favorable predictions of ejected droplet sizes $( \pm 7 \%)$ as well as the critical values of the dimensionless parameters where regime transitions occur. Figure 5.7 provides the numerically computed regime map over a range of $\mathrm{We}_{o}^{1 / 2}$ and $\mathrm{Ca}_{o}$ values. Regime transitions are observed between satellite and mother droplets at $\mathrm{We}_{o}^{1 / 2} \sim 1$ and between mother droplet to ligament ejections at $\mathrm{We}_{o}^{1 / 2} \sim 10$. 
When compared to Fig. 5.2a there is some discrepancy of the regime transitions between the experimental and numerical regime map $( \pm 40 \%)$ although, within the $\mathrm{Su}^{1 / 2}>>1$ regime the transitions occur at a similar order of magnitude. The simulations are carried out only for low viscosity fluids due to the computational difficulty in handling large viscosity differences between the liquid and gas phases. Several runs are performed for comparison with the experiments employing water-glycerol mixtures. Even in such cases the numerics do an adequate job in comparing the order of magnitude of ejected droplet sizes to within $\pm 6 \%$ for perfectly axisymmetric bridge elongation, an example of which is provided in Fig. 5.8 with $U_{o} \sim 0.34 \mathrm{~m} / \mathrm{s}$, $\mathrm{We}_{o}^{1 / 2} \sim 2.5$, and $\mathrm{Ca}_{o} \sim 0.07$. 


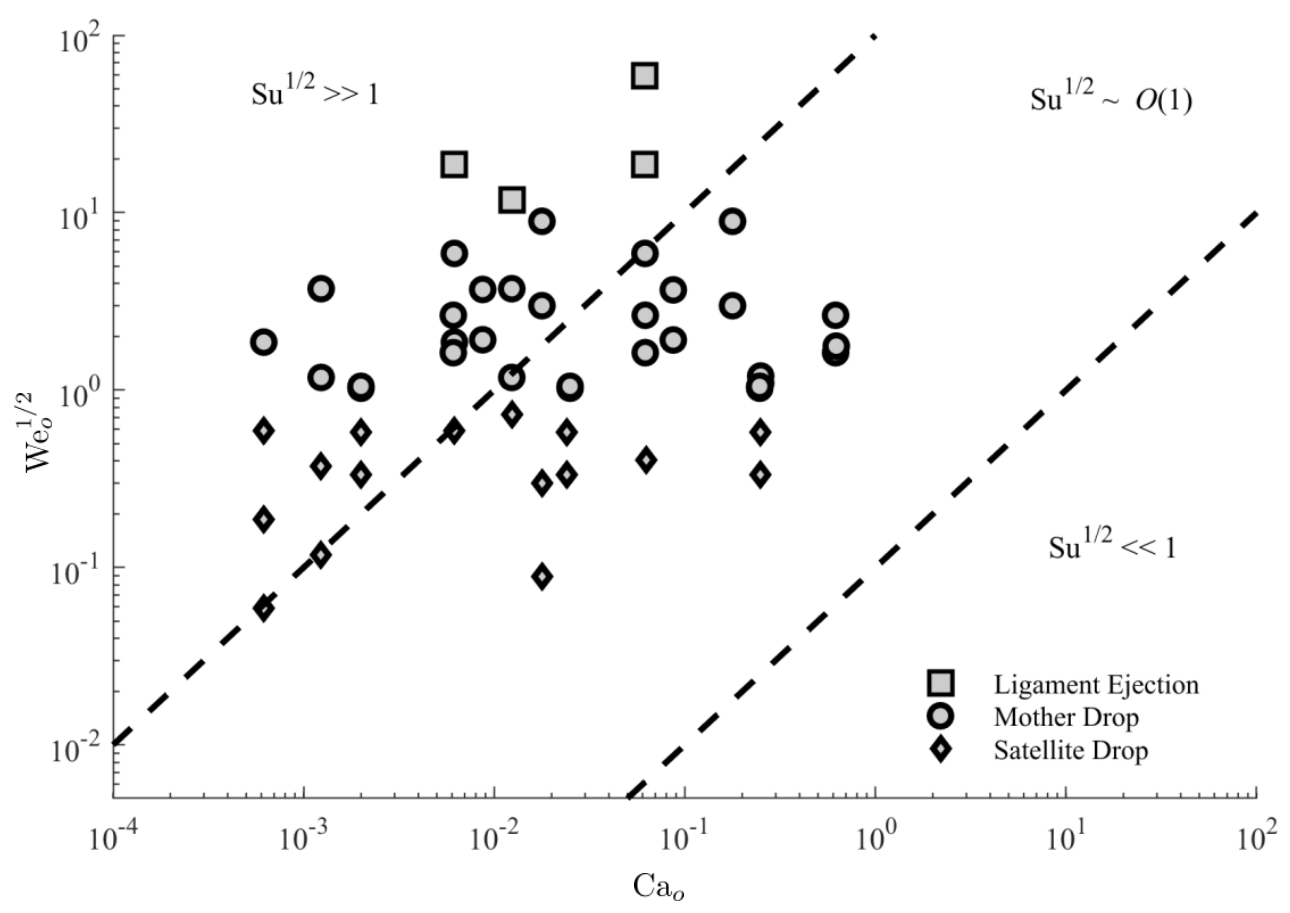

Figure 5.7: Numerically calculated regime map for low- $g$ pipette withdrawals using the open source code Gerris. Regime transitions for the inertial-capillary balance are approximately $\mathrm{We}_{o}^{1 / 2} \sim 1$ for the satellite to mother droplet transition and $\mathrm{We}_{o}^{1 / 2} \sim 10$ for the mother droplet to ligament ejection transition.

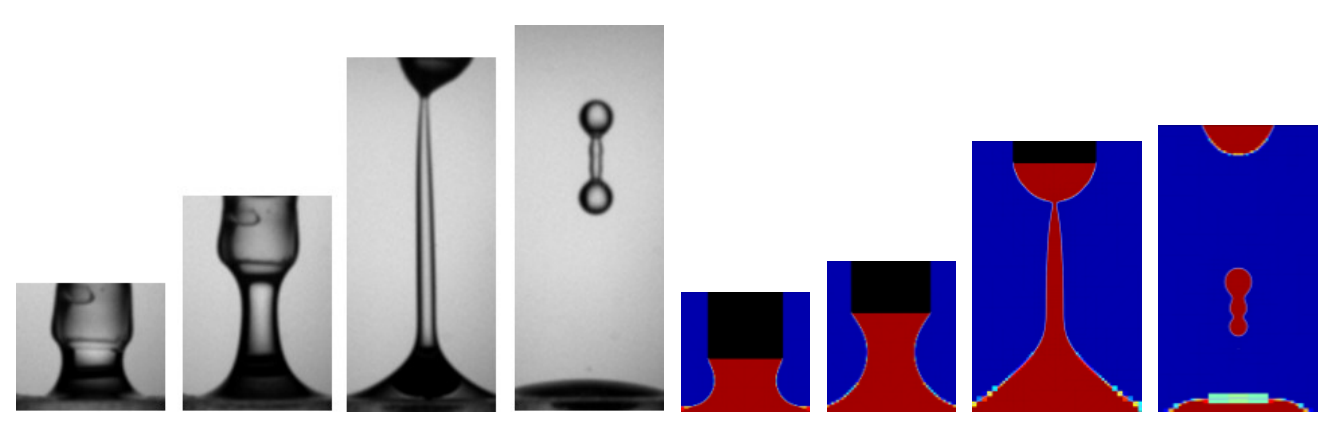

Figure 5.8: A low-g experiment vs. Gerris run for $45.78 \% \mathrm{H}_{2} \mathrm{O} / 54.22 \%$ Glycerol mixture, instrument outer diameter is $3.8 \mathrm{~mm}$ with a relative retraction velocity of $U_{o}=0.34 \mathrm{~m} / \mathrm{s}, \mathrm{Ca}_{o} \sim 0.07$ and $\mathrm{We}_{o}^{1 / 2} \sim 2.5$. The experimental value of $\delta=0.68$ while the computed value is $\delta=0.68$. 


\subsection{Pipette Angle Effects}

Several drop tower experiments are also conducted to asses the contributions of instrument tilt angle $\phi$ to the bridge rupture dynamics and droplet ejections. The tests are conducted maintaining constant retraction velocity $U_{o}$, instrument diameter $2 r_{o}$, and fluid properties with $\phi$ as the only varied parameter. Images from tests are shown in Fig. 5.9 with approximate ejected droplet aspect ratios plotted as a function of $\phi$ in Fig. 5.11. The much larger ligament ejections observed for $\phi>45^{\circ}$ occur due to an increase in the initial volume of these liquid bridges, which is more akin to a liquid sheet. Shown schematically in Fig. 5.10 are the new characteristic dimension $L$ that is the wetted length along the instrument and $L_{c}$ which is a new liquid bridge initial height based on the capillary length scale $L_{c} \sim(\sigma / \rho g)^{1 / 2}$. One could expect that as instrument tilt becomes severe the volume of ejected fluid scales as $V \sim \sigma r_{o} /(2 \rho g \tan \phi)$. The plot in Fig. 5.11 shows ejected droplet radii normalized by $r_{o}$ to highlight the substantial increase in ejected droplet sizes beyond $\phi>45^{\circ}$. As $\phi$ increases, the initial cylindrical configuration of the liquid bridge transitions into more of a sheet of fluid, which in turn produces enormous ejected droplets when compared to the instrument geometry. The added volume of the sheet feeds the liquid bridge during elongation. 


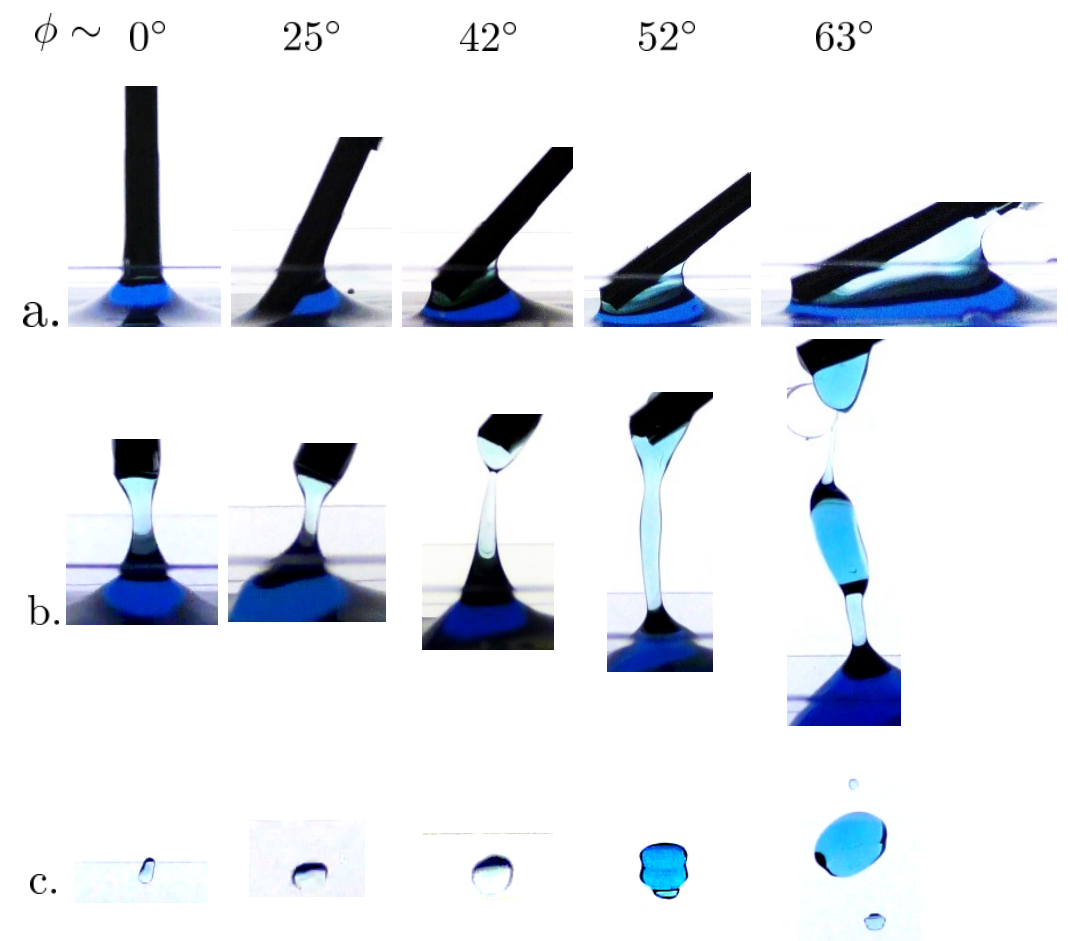

Figure 5.9: Bridge rupture and droplet production for pipette retractions at varying angles, $\phi$ : a. initial wetting condition for each instrument, b. liquid bridge critical height just prior to breakup, and c. ejected droplets produced. A noticeable increase in ejected droplet volume is observed for $\phi>45^{\circ}$.

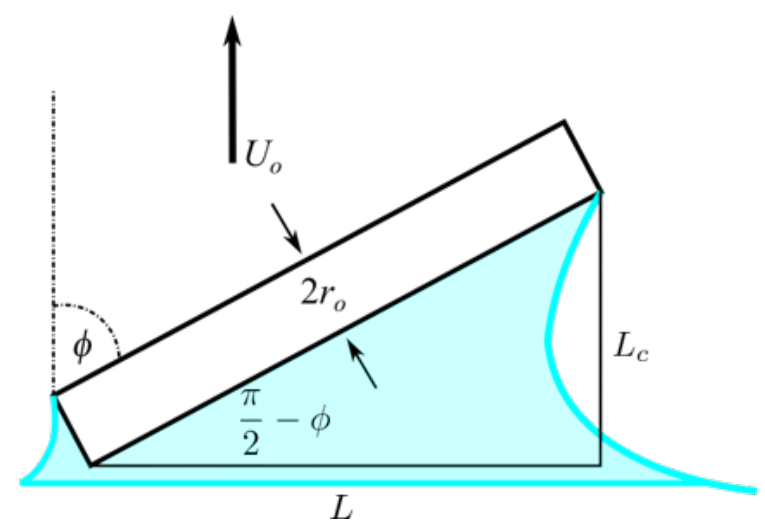

Figure 5.10: Schematic showing the new governing geometry for these large instrument tilt withdrawals. 


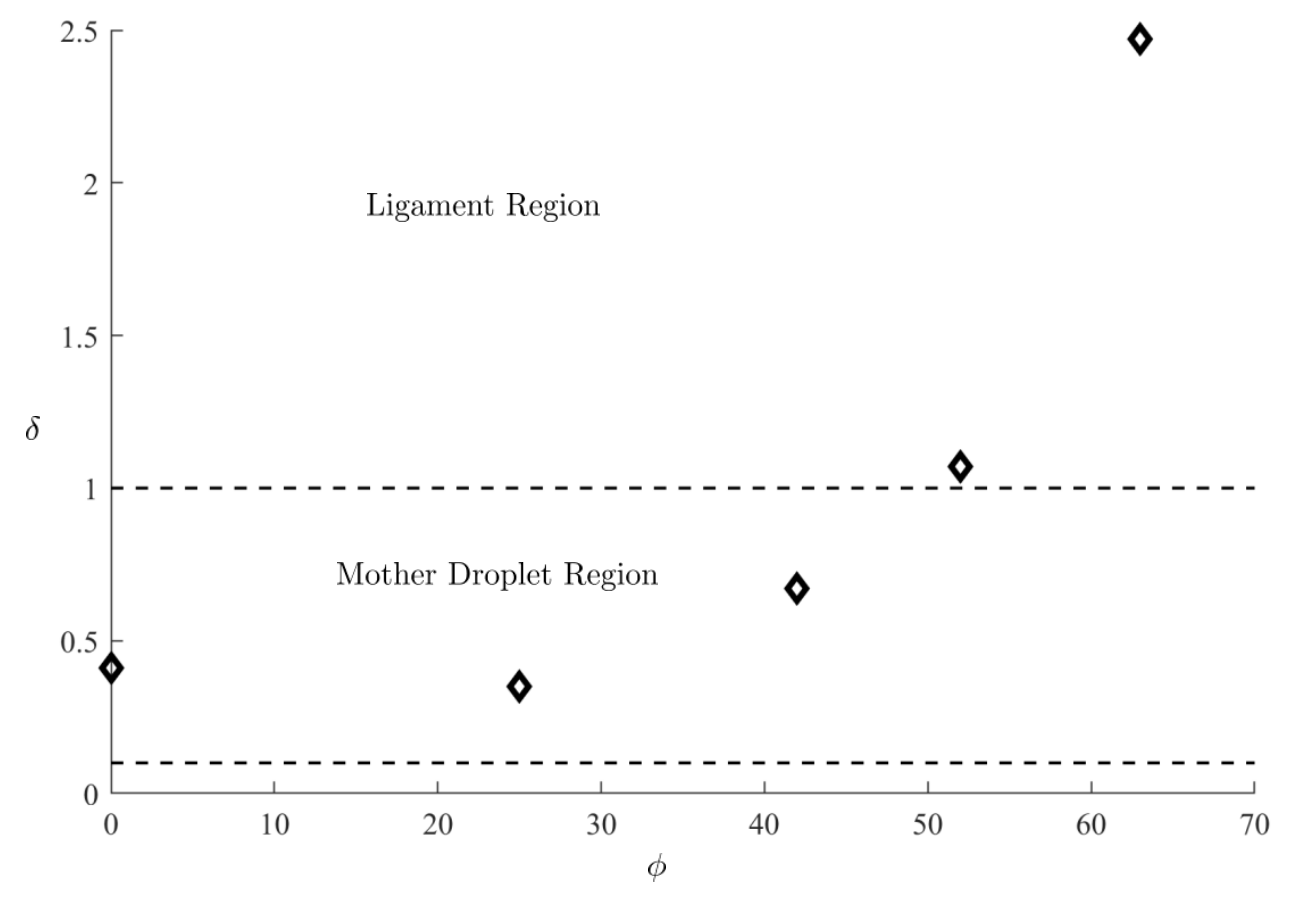

Figure 5.11: The ratio $\delta=r_{d r o p} / r_{o}$ as a function of $\phi$. For $\phi \gtrsim 45^{\circ}$ we observe a transition from 'mother' droplets to large ligament ejections with a substantial increase in ejected fluid volume.

While the complexities of these asymmetric bridge ruptures and droplet ejections are beyond the scope of this work such demonstrations serve as a warning that non axial retractions might bring about larger than expected droplet ejections. 


\section{Chapter 6}

\section{Mitigation Strategies}

\subsection{Introduction}

Figure 6.1 provides three examples of ejected droplet trajectories including direct ejection, ejection and rebound, and ejection with re-coalescence. It is observed that $31 \%$ of droplets are ejected directly upward and away from the instrument/container system into the open environment. The majority of droplets that eject into the reservoir coalesce with it. However $12 \%$ of such droplets rebound from the reservoir free surface up and away from the instrument/reservoir system. The highly variable nature of ejected droplet trajectories necessitates mitigation techniques to safely perform such simple types of wet lab operations in the open environment of spacecraft. 


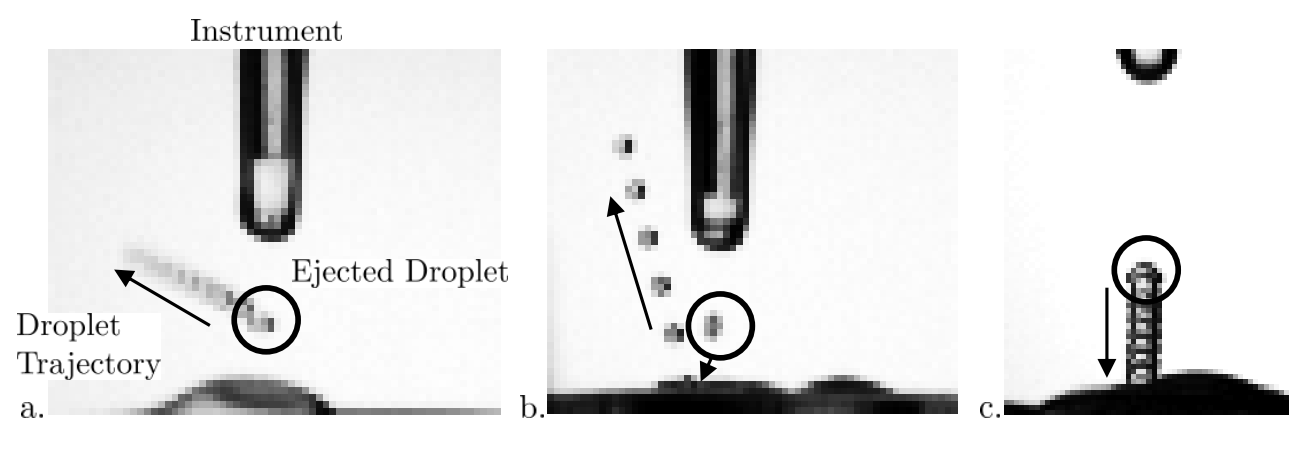

Figure 6.1: Selection of composite images highlighting ejected droplet trajectories: a. direct droplet ejection where the droplet is generated between the instrument and reservoir boundaries with some unknown ejection angle, here $\alpha \sim 60^{\circ}$ from the withdrawal direction. b. ejection and rebound, where the droplet ejects towards the bath and rebounds off the free surface escaping the container, and c. droplet ejected directly into the reservoir free surface, where the droplet re-coalesces back into the bulk fluid.

\subsection{Deep Well Container Geometry}

Velocity vector data for the ejected droplets are collected from the low- $g$ drop tower experiments. As discussed previously, any number of experimental uncertainties and asymmetries can produce these direct ejection vectors for a given ejected droplet, because perfect symmetry leads only to perfectly axial ejections. In general, of the cases analyzed it is observed that approximately $23 \%$ of droplets are ejected upward within a $30^{\circ}$ projection from the rupture location. Simple trigonometric relationships provide expressions that can be used to determine container under-fill levels that provide passive containment for these droplets which impact dry portions of the container, stick, and remain wall-bound. In reference to Fig. 6.2, for a container height of $h_{\text {res }}$ we seek to determine a practical under-fill level that provides ample dry wall area for these ejected droplets to impact and adhere. We note that the ejected 
droplets are produced some height $H F$ above the interface and for a given ejection angle $\alpha$ the droplet must travel a distance $R \tan \alpha$ before it escapes the container. A dimensionless under-fill fraction may be written as a function of mitigation angle,

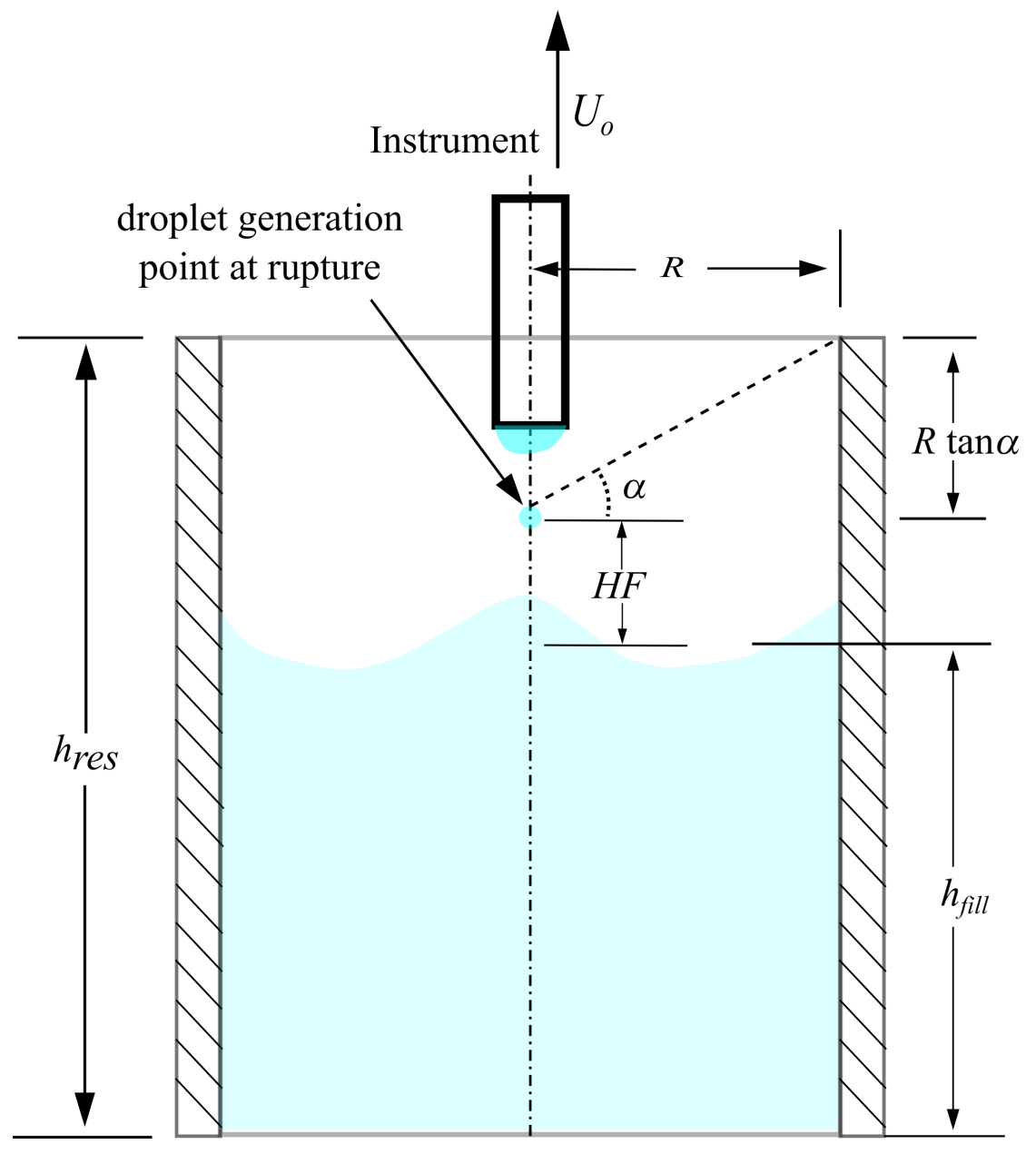

Figure 6.2: Pipette container schematic for deep well mitigation.

$$
h^{*}=1-\left(R^{*} \tan \alpha+H F^{*}\right)
$$




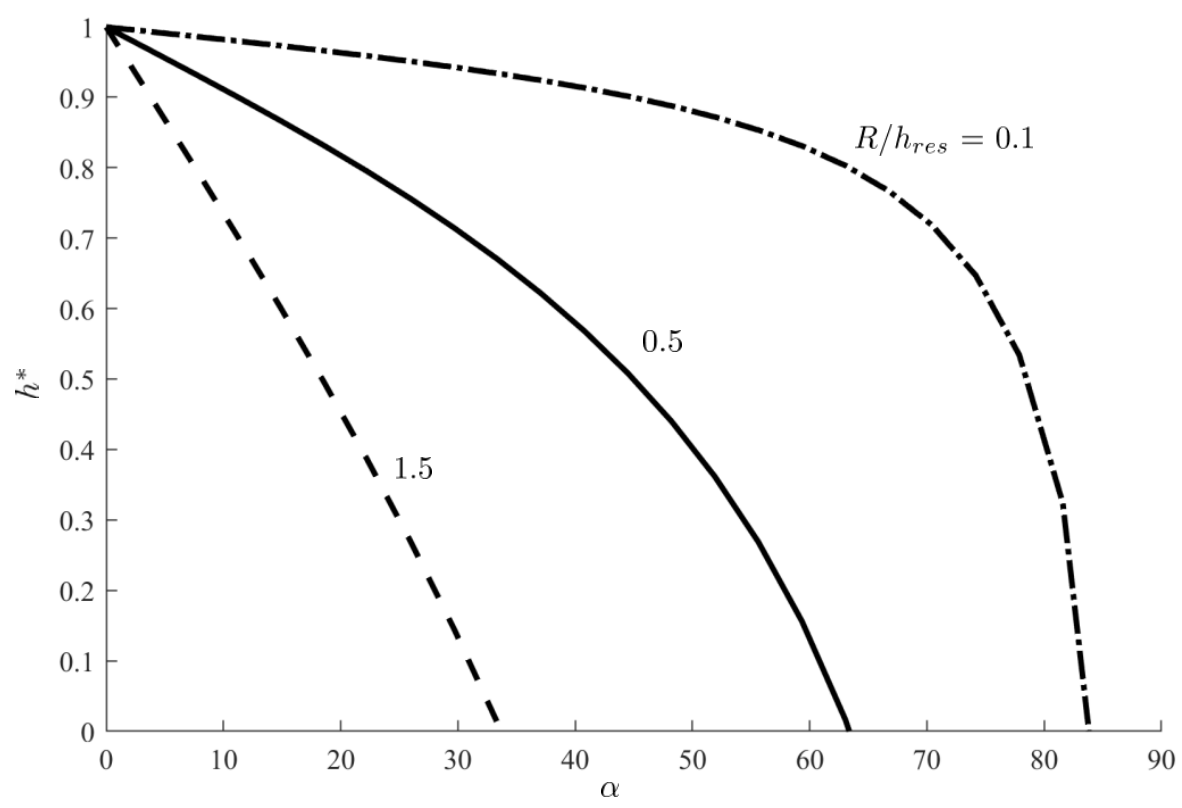

Figure 6.3: Dimensionless fill fraction as a function of mitigation angle $\alpha$ for containers of varying aspect ratios $R / h_{\text {res }}$.

The geometry shown in Fig. 6.2 is a small aspect ratio container with $R / h_{\text {res }}<$ 0.5. Provided in Fig. 6.3 are plots of example fill fractions for multiple container aspect ratios over the domain of possible mitigation angles. For a selected mitigation angle with a container that is under-filled to its corresponding fill fraction one could expect that all droplets ejected with an escape trajectory angle less than the mitigation angle chosen will be contained by the dry portion of the container walls, for example had our experimental containers been under-filled by $20 \%$, guided by Fig. 6.3, we could expect a $10 \%$ improvement in containing the direct ejections observed experimentally. While the tall slender geometries lend to better containment it should be noted that container dimensions may be varied to improve mitigation. For example, tapered containers could provide additional and significant control of 
these ejected droplets by further restricting the area through which these droplets could potentially escape.

\subsection{Air Flow Control}

Similar to the mitigation strategy employed in the STCE demonstrations [10] we seek verification of the ability to contain ejected droplets through the use of a uniform free stream air flow $U_{\infty}$ within the working environment. A schematic of the flow problem is sketched in Figure 6.4 which provides a schematic representation of the the droplet/air flow orientation. Assuming the air flow velocity is higher than the vertical ejection velocity (momentum) we construct force balance equations in the $x$ and $y$ directions in inertial and viscous regimes. Beginning with the $x$ direction we have,

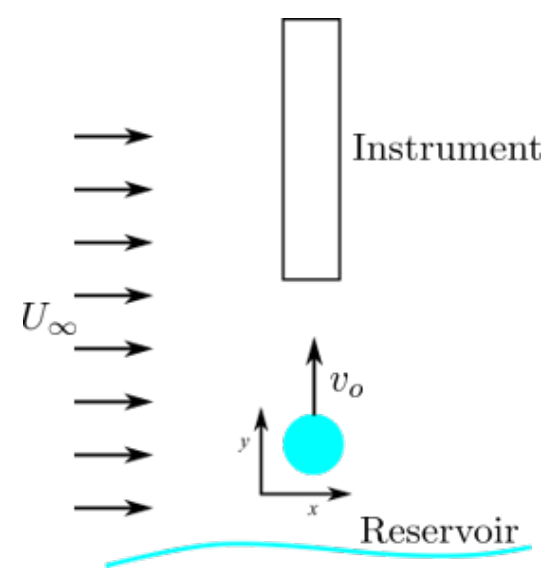

Figure 6.4: Airflow control schematic. A droplet is introduced into a free stream airflow in the $x$-direction. The droplet has an assumed initial velocity component in only the $y$-direction, $v_{o}$. The airflow is confined to some area based on a height in the $y$-direction and some width into the plane.

$$
m \frac{d^{2} x}{d t^{2}}=F_{d r a g_{x}}=\frac{C_{d} \rho_{a} U_{\infty}^{2} A_{a}}{2}
$$


The assumed inertial force acting on the droplet is given as $F_{\text {dragx }}$ with $C_{d}=0.47$, where $m=\pi \rho_{d} D_{d}^{3} / 6$ for an assumed spherical droplet with characteristic diameter of $D_{d}$ and density $\rho_{d}$. Writing the force balance in the $y$-direction gives

$$
m \frac{d^{2} y}{d t^{2}}=F_{d r a g_{y}}=\mu_{a} D_{d} \frac{d y}{d t}
$$

The assumed viscous force acting on the droplet in the $y$ direction is given as $F_{\text {dragy }}$. Using the initial values of $x(t=0)=0, d x / d t(t=0)=0$ and $y(t=0)=0$, and $d y / d t(t=0)=v_{o}$ for the system initial conditions we solve Eq. 6.2 and 6.3 to find

$$
x(t)=0.47 \frac{3 \rho_{a} A_{a} U_{\infty}^{2}}{2 \pi \rho_{d} D_{d}^{3}} t^{2},
$$

and

$$
y(t)=-\frac{v_{o}}{\beta} \exp ^{-\beta t}+\frac{v_{o}}{\beta}
$$

Here $\rho_{a}$ and $\mu_{a}$ are the density and viscosity of air, $A_{a}$ is the cross sectional area of the airflow, $U_{\infty}$ is the air flow velocity, $t$ is time, $v_{o}$ is the droplets initial ejection velocity, with $\beta=6 \mu_{a} /\left(\pi \rho_{d} D_{d}^{2}\right)$.

The experiments appear to be in good agreement with the simple parametric model. Inviscid droplets ejected via pipette operations typically have high frequency oscillations which are assumed spherical in the model.

The ability to apply simple kinematic equations to describe containment methods for these types of droplets lends itself to extensions using other forces. Related work by Schmidt [25] has also established the ability to influence the motion of small volume droplets in low- $g$ using electro-static coulumbic forces demonstrating 
additional options for ejected droplet control.
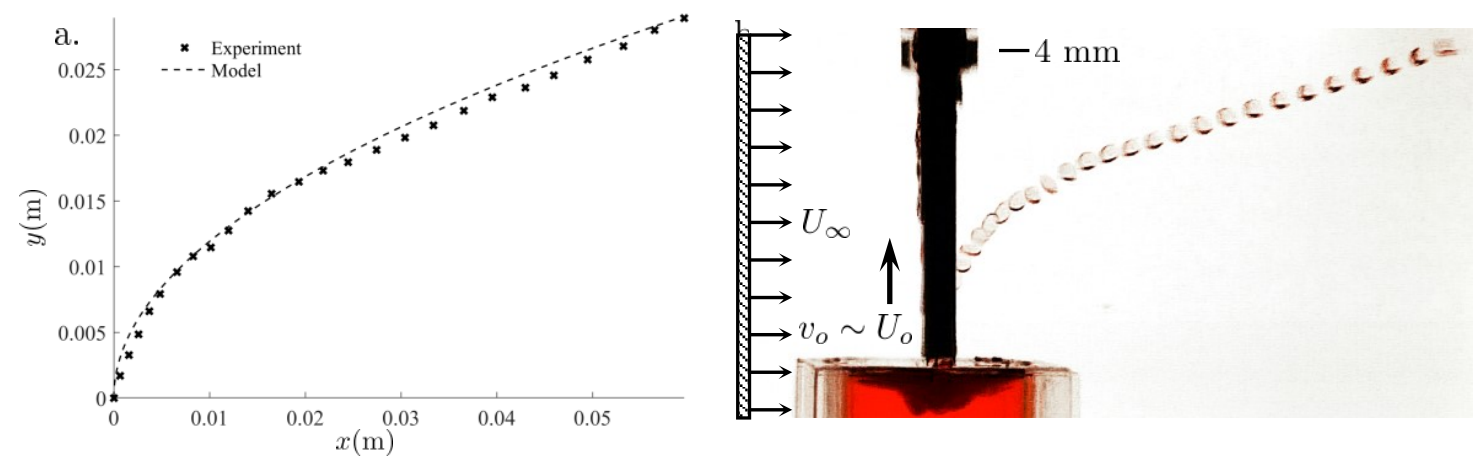

Figure 6.5: a. Eq. 6.4 and 6.5 model compared with data for experiment of ejected droplet in time. b. Composite image from experiment highlighting the effect of airflow containment on droplet trajectories. Droplet initial velocity is assumed straight upward with a magnitude approximately that of $U_{o} \sim 0.5 \mathrm{~m} / \mathrm{s}$. Positioned to the left, out of frame are two vertically stacked $40 \mathrm{~mm}^{2}$ electronics cooling fans blowing air at $\sim 6.9 \mathrm{CFM}\left(U_{\infty} \sim 2.5 \mathrm{~m} / \mathrm{s}\right)$ horizontally rightward.

\subsection{Superhydrophobic Instrument Exterior}

The numerical simulations run using STAR-CCM+ provided a more accurate model than that of Gerris but at the cost of significantly increased computational time. The model still employs a relative motion simplification with the fluid draining away from the instrument at a relative retraction speed $U_{o}$ within a rotationally symmetric domain. The STAR model enables users to model the pipette as a cylindrical annulus to account for fluid within the instrument tip giving a more accurate representation of pipette induced liquid bridge rupture. Yet the effects of this new geometry are negligible when examining the zeroth order ejected droplet sizes produced at rupture. STAR is used to conduct a brief study into effects of instrument exterior wetting conditions on droplet ejections. Figure 6.6 shows the graphical representation of a 
STAR-CCM+ simulation for a $1 \mathrm{~mm}$ OD pipette initially submerged in water. The outer surface of the instrument has a prescribed contact angle $\theta=150^{\circ}$ with an interior surface contact angle of $30^{\circ}$. From the simulation we observe a transition for the liquid bridge's upper boundary characteristic length transitioning from $r_{o}$ to $r_{i}$ as the separation distance between instrument and bulk fluid increases. From the experimental results discussed in Chapter 5, typical ejected droplet dimensions are on average $O\left(r_{o}\right)$ for withdrawal rates akin to manual separations, $0.3 \leq U_{o} \leq 0.5$ $\mathrm{m} / \mathrm{s}$. Provided that instrument geometry has an appreciable wall thickness relative to $r_{o}$, one could expect a decrease in ejected droplet volumes of approximately $\left(r_{i} / r_{o}\right)^{3}$, which are more manageable from a mitigation perspective.

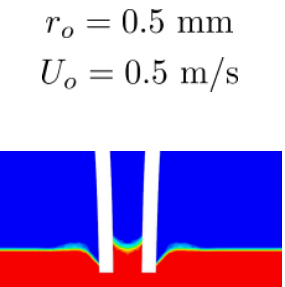

$t=0 \mathrm{~s}$

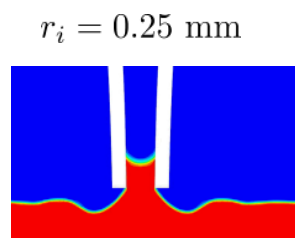

0.001

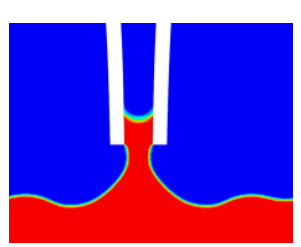

0.002

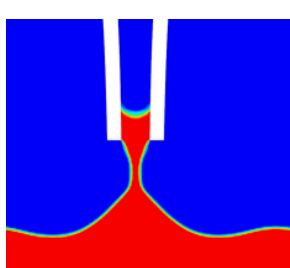

0.0027

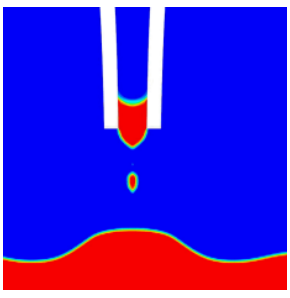

0.004

Figure 6.6: STAR-CCM+ Superhyrophobic instrument tip $\left(r_{o}=0.5 \mathrm{~mm}\right.$, $\left.r_{i}=0.25 \mathrm{~mm}\right)$ simulation with $\theta_{\text {exterior }} \sim 150^{\circ}$ and $\theta_{\text {interior }} \sim 30^{\circ}$ prescribed drain velocity is $0.5 \mathrm{~m} / \mathrm{s}$. Immediate differences between SHS tip and conventional exteriors is the change in liquid bridge upper boundary conditions. The characteristic dimension becomes the instrument inner radii $r_{i}$ rather than $r_{o}$. 


\section{Chapter 7}

\section{Summary \& Future Work}

The work presented herein represents a useful though brief exploration into the fundamental study of liquid bridge elongation leading to rupture akin to standard pipette operations and the subsequent ejection of varying droplet types. We provide experimental and numerical $1-g$ and low- $g$ regime maps for droplet size transitions based on the input parameters discussed in Chapter 2 that are differentiated by input withdrawal parameters and inherent fluid response. The statistics on 3 different observed ejected droplet trajectories are reported. Following the discussion of experimental and numerical observations are two possible mitigation strategies that engineers and designers may employ to reduce contamination possibilities while performing pipette operations in the open environment aboard space craft with an additional numerical study into the effects of instrument wetting conditions on ejected droplet size. While the parameter space of this phenomena is vast we provide several noteworthy conclusions from an operational and design standpoint:

- Rupture typically occurs nearest the smaller boundary: liquid bridge rupture typically occurs first near the instrument then near the free surface. The droplets generated this way typically take on a coalescence ejection trajectory $(\approx 61 \%)$ with a small percentage of those droplets $(\approx 8 \%)$ rebounding and 
subsequently taking an escape trajectory.

- As the instrument radius $r_{o}$ becomes closer to the reservoir radius $R$ the highest order droplet is commonly produced via rupturing the bridge nearly simultaneously at two axial locations. This in turn causes the droplet to travel at very low, manageable speeds.

- Perfect axisymmetric withdrawals produce droplets that are ejected either upwards towards the instrument tip or downwards into the bulk fluid. Experiments suggest $88 \%$ of such droplets re-coalesce, while $12 \%$ rebound and eject away from the pipette/instrument system.

- Asymmetries of the rupture process cause droplets to be ejected at elevated velocities.

- Initial perturbations, asymmetries, non-constant withdrawal rates, and others lead to highly unpredictable behavior in droplet ejection vectors.

- Manual withdrawals with somewhat highly variable withdrawal parameters can be met with external or system mitigation techniques to ensure adequate statistical ejected droplet containment.

- Non axial instrument withdrawals effect the reservoir boundary condition and cause rupture nearest the bulk fluid. This commonly causes the droplets to be ejected upward and away from working areas.

- Pipette wet-lab operations using aqueous solutions will almost always produce a droplet. High viscosity and low withdrawal rates can eliminate such ejections. The impacts of surfactants, visco-elasticity, foams, temperature fields, 
acoustics, $\nabla \sigma$ phenomena, and other effects can also work to eliminate ejections.

- The size and volume of these droplets produced is correlated with instrument size $r_{o}$ and withdrawal rate $U_{o}$.

- Ejected droplet sizes are governed by the parameters of the problem, satellite droplets $\delta<0.2$ occur for $\mathrm{We}_{o}^{1 / 2}<1$, mother droplets $0.2 \leq \delta<1$ occur for $1<\mathrm{We}_{o}^{1 / 2}<5$, and ligament ejections $\delta \geq 1$ occur for $\mathrm{We}_{o}^{1 / 2}>5$, which correspond to the values of $U_{o} / U$ when it is evaluated in the appropriate limits of $\mathrm{Su}^{1 / 2}=\left(\rho r_{o} \sigma / \mu^{2}\right)^{1 / 2}$.

- For conservative estimates designers should expect ejected droplets to be $O\left(r_{o}\right)$ for mitigation techniques.

- The larger the droplet is relative to $r_{o}$ the slower its ejection velocity. Smaller droplets travel at much higher speeds that scale as $U \sim\left(\sigma /\left(\rho r_{o}\right)\right)^{1 / 2}$.

- For non-Newtonian fluids, strain-hardening phenomena acts to stabilize liquid bridges as they undergo elongation. This in turn could result in a larger volume of ejected fluid when rupture inevitably occurs. [26]

- The addition of surfactants, both soluble and insoluble, allow for highly variable liquid bridge dynamics and subsequent droplet ejection. Surfactants act to stabilize liquid bridges through the elongation process in turn allowing for higher volume of fluids to become ejected relative to aqueous counterparts [27].

In conclusion, the experimental parameters explored herein provide insight into inertial-capillary droplet production for typical pipetting geometries and speeds, 
but further work should be pursued to explore corresponding ejection types for $\mathrm{Su}^{1 / 2} \ll 1$, where only 4 data points are available from low- $g$ tests. The asymmetric stable configurations observed in the SE-FIT simulations also merit further attention since the rupture of such bridges is likely to produce equally asymmetric ejections. It is the hope of the author that the work contained herein will serve as a design guide for engineers and scientists to expect, understand, prevent, manage, or contain such fluid ejections, and that such highly efficient fluid unit operations may continue with confidence in outcomes in the open cabin aboard spacecraft. 


\section{Bibliography}

[1] Tomotika S. On the instability of a cylindrical thread of a viscous liquid surrounded by another viscous fluid. Royal Society, 150:322-337, 1935.

[2] Keller J.B., Rubinow S.I., and Tu Y.O. Spatial instability of a jet. 16(12):2052$2055,1973$.

[3] Resnick A., Alexander J., and Iwan D. Plateau Tank Apparatus for The Study of Liquid Bridges. Review of Scientific Instruments, 68:1495-1500, 1998.

[4] Meseguer J, Sanz A, and Lopez J. Liquid bridge breakages aboard spacelab-d1. Journal of Crystal Growth, 78(2):325 - 334, 1986.

[5] Tjahjadi M., Stone H.A., and Ottino J.M. Satellite and Subsatellite Formation in Capillary Breakup. Journal of Fluid Mechanics, 243:297-317, 1992.

[6] Dodds S. and Carvalho M. Stretching liquid bridges with moving conatact lines: The role of inertia. Physics of Fluids, 23:1-11, 2011.

[7] Marmottant P. and Villermaux E. Fragmentation of Stretched Liquid Ligaments. Physics of Fluids, 16(8):2732-2741, 2004.

[8] Wollman A.P. Large Length Scale Capillary Fluidics: From jumping Bubbles to Drinking in Space. Ph.d. dissertation, Portland State University, June 2016. 
[9] Kristen K.J. Biomolecule sequencer experiment. https://www.nasa.gov/ mission_pages/station/research/news/biomolecule_sequencer, 2016.

[10] Turner C., Goodman J., Mohler S., Mungin R., and Weislogel M. Mitigation of micro-droplet ejections during open cabin unit operations aboard iss. In 49th International Conference on Environmental Systems, 201, pages 1-8, July 2019.

[11] Weislogel M.M. and Machles A. Slime in space. https://www.space.com/ nickelodeon-slime-launching-to-space.html, 2020.

[12] Smolka L.B. and Belmonte A. Drop pinch-off and filament dynamics of wormlike micellar fluids. Jounral of Non-Newtonian Fluid Mechanics, 115:1-25, 2003.

[13] Basaran O., Zhang X., and Padgett R. Nonlinear deformation and breakup of stretching liquid bridges. Journal of Fluid Mechanics, 329:207-245, 1996.

[14] Eggers J. Nonlinear dynamics and breakup of free-surface flows. Reviews of Modern Physics, 69(1833):865-929, 1997.

[15] Weislogel M.M., Chen Y., and Bolleddula D. A better nondimensionalization scheme for slender laminar flows: The laplacian operator scaling method. Physics of Fluids, 20(9):093602, 2008.

[16] Wollman A.P., Weislogel M.M., Wiles B., and Pettit D. More investigations in capillary fluidics using a drop tower. Experiments in Fluids, 57(4):1-17, 2016.

[17] Schindelin J., Arganda carreras I., Frise E., Kaynig V., Pietzsch T., Preibisch S., Rueden C., Saalfeld S., Schmid B., Tinevez J., White D.J., Hartenstein V., 
Tomancak P., and Cardona A. Fiji - an Open Source platform for biological image analysis. Nature Methods, 9(7):676-682, 2019.

[18] Chen Y., Schaeffer B.M., and Weislogel M.M. Introducing SE-FIT:Surface Evolver - Fluid Interface Tool for Studying Capillary Surfaces. Number January, pages 1-11, 2011.

[19] Brakke K.A. The surface evolver. Experimental Mathematics, 1(2):141-165, 1992.

[20] Popinet S. An accurate adaptive solver for surface-tension-driven interfacial flows. Journal of Computational Physics, 228(16):5838-5866, 2009.

[21] Popinet S. Gerris : a tree-based adaptive solver for the incompressible Euler equations in complex geometries. Journal of Computational Physics, 190:572600, 2003.

[22] Fawehinmi O.B. A combined experimental and computational fluid dynamics analysis of the dynamics of drop formation. 219:933-947, 2005.

[23] Zhuang J. and Ju Y.S. A Combined Experimental and Numerical Modeling Study of the Deformation and Rupture of Axisymmetric Liquid Bridges under Coaxial Stretching. The ACS Journal of Surfaces and Colloids, 37:1-10, 2015.

[24] Viestenz K.J., Jenson R.M., Weislogel M.M., and Sargusingh M.J. Capillary structures for exploration life support payload experiment. In 48th International Conference on Environmental Systems, volume ICES-2018-241, pages 1-11, July 2018. 
[25] Schmidt E.S. Electro-Drop Bouncing in Low-Gravity by. Master's thesis, Portland State University, 2018.

[26] McKinley G.H. Spiegelberg S.H. The extensional rheology of non-newtonian materials. In Third Microgravity Fluid Physics Conference, pages 377-382, 1996.

[27] Montanero J.M. Review on the dynamics of isothermal liquid bridges. Applied Mechanics Review, 72:1-38, August 2019. 


\section{Appendix A: Experimental Parameters}

Table A.1: Low- $g$ experimental parameters for pipette droplet ejection study.

\begin{tabular}{|c|c|c|c|c|c|c|}
\hline No. & $2 r_{o}(\mathrm{~mm})$ & $2 R(\mathrm{~mm})$ & $U_{o}(\mathrm{~m} / \mathrm{s})$ & $\mu(\mathrm{kg} /(\mathrm{m} \cdot \mathrm{s}))$ & $\rho\left(\mathrm{kg} / \mathrm{m}^{3}\right)$ & $\sigma(\mathrm{mN} / \mathrm{m}) \pm 0.2$ \\
\hline 1 & 1 & 2 & 0.03 & 0.00095 & 997.61 & 69.1 \\
\hline 2 & 1 & 4 & 0.02 & 0.00095 & 997.61 & 69.1 \\
\hline 3 & 1 & 50 & 0.02 & 0.00095 & 997.61 & 69.1 \\
\hline 4 & 1 & 2 & 0.46 & 0.00095 & 997.61 & 69.1 \\
\hline 5 & 1 & 2 & 0.4 & 0.01 & 1154.7 & 62.8 \\
\hline 6 & 1 & 4 & 0.5 & 0.00095 & 997.61 & 69.1 \\
\hline 7 & 1 & 4 & 0.41 & 0.01 & 1154.7 & 62.8 \\
\hline 8 & 1 & 6 & 0.43 & 0.00095 & 997.61 & 69.1 \\
\hline 9 & 1 & 6 & 0.51 & 0.01 & 1154.7 & 62.8 \\
\hline 10 & 1 & 10 & 0.02 & 0.00095 & 997.61 & 69.1 \\
\hline 11 & 1 & 10 & 0.59 & 0.00095 & 997.61 & 69.1 \\
\hline 12 & 1 & 10 & 0.43 & 0.01 & 1154.7 & 62.8 \\
\hline 13 & 1 & 20 & 0.53 & 0.00095 & 997.61 & 69.1 \\
\hline 14 & 1 & 20 & 0.53 & 0.01 & 1154.7 & 62.8 \\
\hline 15 & 3.6 & 4 & 0.45 & 0.00095 & 997.61 & 69.1 \\
\hline 16 & 1 & 6 & 0.02 & 0.00095 & 997.61 & 69.1 \\
\hline 17 & 1 & 10 & 0.03 & 0.00095 & 997.61 & 69.1 \\
\hline 18 & 1 & 20 & 0.03 & 0.00095 & 997.61 & 69.1 \\
\hline 19 & 1 & 20 & 0.02 & 0.08 & 1216 & 66.5 \\
\hline 20 & 3.6 & 4 & 0.41 & 0.00095 & 997.61 & 69.1 \\
\hline 21 & 3.6 & 6 & 0.43 & 0.01 & 1154.7 & 62.8 \\
\hline 22 & 3.6 & 6 & 0.49 & 0.00095 & 997.61 & 69.1 \\
\hline
\end{tabular}


Table A.1: Low- $g$ experimental parameters for pipette droplet ejection study.

\begin{tabular}{|c|c|c|c|c|c|c|}
\hline No. & $2 r_{o}(\mathrm{~mm})$ & $2 R(\mathrm{~mm})$ & $U_{o}(\mathrm{~m} / \mathrm{s})$ & $\mu(\mathrm{kg} /(\mathrm{m} \cdot \mathrm{s}))$ & $\rho\left(\mathrm{kg} / \mathrm{m}^{3}\right)$ & $\sigma(\mathrm{mN} / \mathrm{m}) \pm 0.2$ \\
\hline 23 & 4 & 6 & 0.03 & 0.00095 & 997.61 & 69.1 \\
\hline 24 & 4 & 10 & 0.42 & 0.00095 & 997.61 & 69.1 \\
\hline 25 & 4 & 4 & 0.03 & 0.00095 & 997.61 & 69.1 \\
\hline 26 & 3.6 & 20 & 0.02 & 0.00095 & 997.61 & 69.1 \\
\hline 27 & 3.6 & 20 & 0.02 & 0.00095 & 997.61 & 69.1 \\
\hline 28 & 4 & 10 & 0.02 & 0.00095 & 997.61 & 69.1 \\
\hline 29 & 3.6 & 20 & 0.51 & 0.01 & 1154.7 & 62.8 \\
\hline 30 & 3.6 & 10 & 0.54 & 0.01 & 1154.7 & 62.8 \\
\hline 31 & 4 & 6 & 0.56 & 0.01 & 1154.7 & 62.8 \\
\hline 32 & 1 & 2 & 0.48 & 0.08 & 1216 & 66.5 \\
\hline 33 & 1 & 4 & 0.15 & 0.08 & 1216 & 66.5 \\
\hline 34 & 1 & 6 & 0.44 & 0.08 & 1216 & 66.5 \\
\hline 35 & 1.69 & 20 & 0.6 & 0.08 & 1216 & 66.5 \\
\hline 36 & 1 & 10 & 0.84 & 0.00095 & 997.61 & 69.1 \\
\hline 37 & 1 & 20 & 0.96 & 0.00095 & 997.61 & 69.1 \\
\hline 38 & 3.6 & 20 & 1 & 0.00095 & 997.61 & 69.1 \\
\hline 39 & 3.6 & 10 & 1.23 & 0.00095 & 997.61 & 69.1 \\
\hline 40 & 3.6 & 10 & 1.23 & 0.00095 & 997.61 & 69.1 \\
\hline 41 & 4 & 20 & 1.16 & 0.00095 & 997.61 & 69.1 \\
\hline 42 & 4 & 6 & 1.1 & 0.00095 & 997.61 & 69.1 \\
\hline 43 & 1 & 6 & 0.83 & 0.00095 & 997.61 & 69.1 \\
\hline 44 & 2.1 & 6 & 0.86 & 0.00095 & 997.61 & 69.1 \\
\hline 45 & 2.1 & 6 & 0.59 & 0.00095 & 997.61 & 69.1 \\
\hline
\end{tabular}


Table A.1: Low- $g$ experimental parameters for pipette droplet ejection study.

\begin{tabular}{|c|c|c|c|c|c|c|}
\hline No. & $2 r_{o}(\mathrm{~mm})$ & $2 R(\mathrm{~mm})$ & $U_{o}(\mathrm{~m} / \mathrm{s})$ & $\mu(\mathrm{kg} /(\mathrm{m} \cdot \mathrm{s}))$ & $\rho\left(\mathrm{kg} / \mathrm{m}^{3}\right)$ & $\sigma(\mathrm{mN} / \mathrm{m}) \pm 0.2$ \\
\hline 46 & 3.6 & 20 & 0.99 & 0.00095 & 997.61 & 69.1 \\
\hline 47 & 1 & 20 & 0.02 & 0.01 & 1154.7 & 62.8 \\
\hline 48 & 2.1 & 20 & 0.02 & 0.01 & 1154.7 & 62.8 \\
\hline 49 & 4 & 20 & 0.02 & 0.01 & 1154.7 & 62.8 \\
\hline 50 & 1 & 20 & 0.02 & 0.08 & 1216 & 66.5 \\
\hline 51 & 2.1 & 20 & 0.02 & 0.08 & 1216 & 66.5 \\
\hline 52 & 4 & 50 & 0.02 & 0.08 & 1216 & 66.5 \\
\hline 53 & 3.35 & 50 & 0.25 & 0.00095 & 997.61 & 69.1 \\
\hline 54 & 3.35 & 50 & 0.32 & 0.00095 & 997.61 & 69.1 \\
\hline 55 & 3.35 & 50 & 0.2 & 0.00095 & 997.61 & 69.1 \\
\hline 56 & 3.35 & 50 & 0.23 & 0.00095 & 997.61 & 69.1 \\
\hline 57 & 3.35 & 50 & 0.43 & 0.00095 & 997.61 & 69.1 \\
\hline 58 & 3.35 & 50 & 0.5 & 0.00095 & 997.61 & 69.1 \\
\hline 59 & 3.35 & 50 & 0.52 & 0.00095 & 997.61 & 69.1 \\
\hline 60 & 3.35 & 50 & 0.6 & 0.00095 & 997.61 & 69.1 \\
\hline 61 & 3.35 & 50 & 0.69 & 0.00095 & 997.61 & 69.1 \\
\hline 62 & 3.35 & 50 & 0.72 & 0.00095 & 997.61 & 69.1 \\
\hline 63 & 3.35 & 50 & 0.66 & 0.00095 & 997.61 & 69.1 \\
\hline 64 & 3.35 & 50 & 0.56 & 0.00095 & 997.61 & 69.1 \\
\hline 65 & 1 & 50 & 0.2 & 0.00095 & 997.61 & 69.1 \\
\hline 66 & 2.75 & 50 & 0.2 & 0.00095 & 997.61 & 69.1 \\
\hline 67 & 3.41 & 50 & 0.2 & 0.00095 & 997.61 & 69.1 \\
\hline 68 & 4 & 50 & 0.2 & 0.00095 & 997.61 & 69.1 \\
\hline
\end{tabular}


Table A.1: Low- $g$ experimental parameters for pipette droplet ejection study.

\begin{tabular}{|c|c|c|c|c|c|c|}
\hline No. & $2 r_{o}(\mathrm{~mm})$ & $2 R(\mathrm{~mm})$ & $U_{o}(\mathrm{~m} / \mathrm{s})$ & $\mu(\mathrm{kg} /(\mathrm{m} \cdot \mathrm{s}))$ & $\rho\left(\mathrm{kg} / \mathrm{m}^{3}\right)$ & $\sigma(\mathrm{mN} / \mathrm{m}) \pm 0.2$ \\
\hline 69 & 2.55 & 50 & 0.26 & 0.00095 & 997.61 & 69.1 \\
\hline 70 & 2.75 & 50 & 0.19 & 0.00095 & 997.61 & 69.1 \\
\hline 71 & 3.41 & 50 & 0.19 & 0.00095 & 997.61 & 69.1 \\
\hline 72 & 4 & 10 & 0.19 & 0.00095 & 997.61 & 69.1 \\
\hline 73 & 3 & 10 & 0.24 & 0.08 & 1216 & 66.5 \\
\hline 74 & 2.75 & 10 & 0.24 & 0.08 & 1216 & 66.5 \\
\hline 75 & 3.41 & 10 & 0.24 & 0.08 & 1216 & 66.5 \\
\hline 76 & 4 & 10 & 0.24 & 0.08 & 1216 & 66.5 \\
\hline 77 & 1 & 10 & 0.17 & 0.08 & 1216 & 66.5 \\
\hline 78 & 2.75 & 10 & 0.17 & 0.08 & 1216 & 66.5 \\
\hline 79 & 3.41 & 10 & 0.17 & 0.08 & 1216 & 66.5 \\
\hline 80 & 4 & 10 & 0.17 & 0.08 & 1216 & 66.5 \\
\hline 81 & 1 & 10 & 0.1 & 0.68 & 1253.8 & 64.2 \\
\hline 82 & 2.75 & 10 & 0.1 & 0.68 & 1253.8 & 64.2 \\
\hline 83 & 3.41 & 10 & 0.1 & 0.68 & 1253.8 & 64.2 \\
\hline 84 & 4 & 10 & 0.1 & 0.68 & 1253.8 & 64.2 \\
\hline 85 & 1 & 10 & 0.11 & 0.08 & 1216 & 66.5 \\
\hline 86 & 2.75 & 10 & 0.11 & 0.08 & 1216 & 66.5 \\
\hline 87 & 3.41 & 10 & 0.11 & 0.08 & 1216 & 66.5 \\
\hline 88 & 4 & 10 & 0.11 & 0.08 & 1216 & 66.5 \\
\hline 89 & 1 & 10 & 0.29 & 0.68 & 1253.8 & 64.2 \\
\hline 90 & 2.75 & 10 & 0.29 & 0.68 & 1253.8 & 64.2 \\
\hline 91 & 3.41 & 10 & 0.29 & 0.68 & 1253.8 & 64.2 \\
\hline
\end{tabular}


Table A.1: Low- $g$ experimental parameters for pipette droplet ejection study.

\begin{tabular}{ccccccc} 
No. & $2 r_{o}(\mathrm{~mm})$ & $2 R(\mathrm{~mm})$ & $U_{o}(\mathrm{~m} / \mathrm{s})$ & $\mu(\mathrm{kg} /(\mathrm{m} \cdot \mathrm{s}))$ & $\rho\left(\mathrm{kg} / \mathrm{m}^{3}\right)$ & $\sigma(\mathrm{mN} / \mathrm{m}) \pm 0.2$ \\
\hline 92 & 4 & 70 & 0.29 & 0.68 & 1253.8 & 64.2 \\
93 & 13.65 & NA & 0.28 & 0.00095 & 997.61 & 69.1 \\
94 & 125 & NA & 0.01 & 200 & 997.61 & 69.1 \\
95 & 125 & NA & 0.29 & 200 & 997.61 & 69.1 \\
96 & 125 & NA & 0.37 & 200 & 997.61 & 69.1 \\
97 & 125 & NA & 0.5 & 200 & 997.61 & 69.1 \\
98 & 125 & NA & 0.8 & 200 & 997.61 & 69.1
\end{tabular}

Table A.2: Dimensionless groups for low- $g$ pipette droplet ejection study.

\begin{tabular}{cccccc} 
No. & $\mathrm{Su}$ & $\mathrm{Ca}_{o}$ & $\mathrm{We}_{o}^{1 / 2}$ & $U_{o} / U$ & $\delta$ \\
\hline 1 & 39889 & 0.0004 & 0.08 & 0.1 & $\mathrm{NA}$ \\
2 & 39889 & 0.0003 & 0.06 & 0.1 & $\mathrm{NA}$ \\
3 & 39889 & 0.0002 & 0.05 & 0.05 & $\mathrm{NA}$ \\
4 & 39889 & 0.0061 & 1.21 & 1.2 & 0.4 \\
5 & 360 & 0.06 & 1.05 & 1.1 & 0.1 \\
6 & 39889 & 0.007 & 1.32 & 1.3 & 0.3 \\
7 & 360 & 0.06 & 1.08 & 1.1 & 0.6 \\
8 & 39889 & 0.006 & 1.13 & 1.1 & 0.5 \\
9 & 360 & 0.07 & 1.34 & 1.4 & 0.96 \\
10 & 39889 & 0.0002 & 0.04 & 0.04 & $\mathrm{NA}$ \\
11 & 39889 & 0.008 & 1.55 & 1.6 & 0.7 \\
12 & 360 & 0.06 & 1.13 & 1.2 & 0.8 \\
13 & 39889 & 0.007 & 1.40 & 1.4 & 0.49
\end{tabular}


Table A.2: Dimensionless groups for low- $g$ pipette droplet ejection study.

\begin{tabular}{|c|c|c|c|c|c|}
\hline No. & $\mathrm{Su}$ & $\mathrm{Ca}_{o}$ & $\mathrm{We}_{o}^{1 / 2}$ & $U_{o} / U$ & $\delta$ \\
\hline 14 & 360 & 0.07 & 1.40 & 1.4 & 1.1 \\
\hline 15 & 143601 & 0.006 & 2.25 & 2.3 & 0.3 \\
\hline 16 & 39889 & 0.0003 & 0.06 & 0.1 & $\mathrm{NA}$ \\
\hline 17 & 39889 & 0.0003 & 0.07 & 0.1 & NA \\
\hline 18 & 39889 & 0.0004 & 0.07 & 0.1 & $\mathrm{NA}$ \\
\hline 19 & 6 & 0.02 & 0.06 & 0.1 & 0.13 \\
\hline 20 & 143601 & 0.005 & 2.05 & 2.1 & 0.3 \\
\hline 21 & 1296 & 0.06 & 2.15 & 2.2 & 0.3 \\
\hline 22 & 143601 & 0.006 & 2.45 & 2.5 & 0.5 \\
\hline 23 & 159557 & 0.0003 & 0.13 & 0.1 & $\mathrm{NA}$ \\
\hline 24 & 159557 & 0.006 & 2.21 & 2.2 & 0.4 \\
\hline 25 & 159557 & 0.0004 & 0.14 & 0.1 & 0.1 \\
\hline 26 & 143601 & 0.0003 & 0.11 & 0.1 & 0.1 \\
\hline 27 & 143601 & 0.0003 & 0.12 & 0.1 & 0.2 \\
\hline 28 & 159557 & 0.0003 & 0.13 & 0.1 & 0.2 \\
\hline 29 & 1296 & 0.07 & 2.55 & 2.6 & 0.7 \\
\hline 30 & 1296 & 0.08 & 2.70 & 2.7 & 0.7 \\
\hline 31 & 1440 & 0.08 & 2.95 & 3.0 & 0.6 \\
\hline 32 & 6 & 0.5 & 1.26 & 1.6 & 0.6 \\
\hline 33 & 6 & 0.17 & 0.40 & 0.5 & 0.2 \\
\hline 34 & 6 & 0.49 & 1.16 & 1.4 & 0.4 \\
\hline 35 & 10 & 0.67 & 2.06 & 2.4 & 1.2 \\
\hline 36 & 39889 & 0.011 & 2.21 & 2.2 & 0.5 \\
\hline
\end{tabular}


Table A.2: Dimensionless groups for low-g pipette droplet ejection study.

\begin{tabular}{cccccc} 
No. & $\mathrm{Su}$ & $\mathrm{Ca}_{o}$ & $\mathrm{We}_{o}^{1 / 2}$ & $U_{o} / U$ & $\delta$ \\
\hline 37 & 39889 & 0.013 & 2.53 & 2.5 & 0.8 \\
38 & 143601 & 0.013 & 5.00 & 5.0 & 1.2 \\
39 & 143601 & 0.016 & 6.15 & 6.2 & 1.3 \\
40 & 143601 & 0.016 & 6.15 & 6.2 & 1.2 \\
41 & 159557 & 0.015 & 6.11 & 6.1 & 1.1 \\
42 & 159557 & 0.015 & 5.80 & 5.8 & 1.2 \\
43 & 39889 & 0.011 & 2.19 & 2.2 & 0.7 \\
44 & 83767 & 0.011 & 3.28 & 3.3 & 0.5 \\
45 & 83767 & 0.008 & 2.25 & 2.3 & 0.5 \\
46 & 143601 & 0.013 & 4.95 & 5.0 & 0.5 \\
47 & 360 & 0.003 & 0.05 & 0.05 & $\mathrm{NA}$ \\
48 & 756 & 0.003 & 0.07 & 0.1 & 0.1 \\
49 & 1440 & 0.002 & 0.09 & 0.1 & 0.1 \\
50 & 6 & 0.022 & 0.05 & 0.1 & 0.1 \\
51 & 12 & 0.018 & 0.06 & 0.1 & 0.07 \\
52 & 23 & 0.018 & 0.08 & 0.1 & 0.08 \\
53 & 133629 & 0.003 & 1.21 & 1.2 & 0.2 \\
54 & 133629 & 0.004 & 1.54 & 1.5 & 0.3 \\
55 & 133629 & 0.003 & 0.96 & 1.0 & 0.6 \\
56 & 133629 & 0.003 & 1.11 & 1.1 & 0.4 \\
57 & 133629 & 0.006 & 2.07 & 2.1 & 0.4 \\
58 & 133629 & 0.007 & 2.41 & 2.4 & 0.4 \\
& 133629 & 0.007 & 2.51 & 2.5 & 0.5
\end{tabular}


Table A.2: Dimensionless groups for low- $g$ pipette droplet ejection study.

\begin{tabular}{cccccc} 
No. & $\mathrm{Su}$ & $\mathrm{Ca}_{o}$ & $\mathrm{We}_{o}^{1 / 2}$ & $U_{o} / U$ & $\delta$ \\
\hline 60 & 133629 & 0.008 & 2.89 & 2.9 & 0.6 \\
61 & 133629 & 0.009 & 3.33 & 3.3 & 0.5 \\
62 & 133629 & 0.01 & 3.47 & 3.5 & 0.5 \\
63 & 133629 & 0.009 & 3.18 & 3.2 & 0.5 \\
64 & 133629 & 0.007 & 2.70 & 2.7 & 0.6 \\
65 & 39889 & 0.003 & 0.53 & 0.5 & $\mathrm{NA}$ \\
66 & 109695 & 0.003 & 0.87 & 0.9 & 0.4 \\
67 & 136022 & 0.003 & 0.97 & 1.0 & 0.3 \\
68 & 159557 & 0.003 & 1.05 & 1.1 & 0.4 \\
69 & 1017175 & 0.003 & 3.46 & 3.5 & 0.5 \\
70 & 109695 & 0.003 & 0.83 & 0.8 & 0.3 \\
71 & 136022 & 0.003 & 0.92 & 0.9 & 0.3 \\
72 & 159557 & 0.003 & 1.00 & 1.0 & 0.3 \\
73 & 17 & 0.27 & 1.10 & 1.2 & 0.6 \\
74 & 15 & 0.27 & 1.05 & 1.2 & 0.8 \\
75 & 19 & 0.27 & 1.17 & 1.3 & 0.6 \\
76 & 23 & 0.27 & 1.26 & 1.4 & 0.6 \\
77 & 6 & 0.18 & 0.43 & 0.5 & 0.7 \\
78 & 15 & 0.18 & 0.72 & 0.8 & 0.6 \\
79 & 19 & 0.18 & 0.80 & 0.9 & 0.6 \\
80 & 23 & 0.18 & 0.87 & 0.3 & 0.4 \\
75 & 0.36 & 0.26 & 0.5 & $\mathrm{NA}$ \\
75 & 0.36 & 0.44 & 0.7 & 0.2
\end{tabular}


Table A.2: Dimensionless groups for low- $g$ pipette droplet ejection study.

\begin{tabular}{cccccc} 
No. & $\mathrm{Su}$ & $\mathrm{Ca}_{o}$ & $\mathrm{We}_{o}^{1 / 2}$ & $U_{o} / U$ & $\delta$ \\
\hline 83 & 2 & 0.36 & 0.49 & 0.7 & 0.2 \\
84 & 2 & 0.36 & 0.53 & 0.7 & 0.2 \\
85 & 6 & 0.12 & 0.29 & 0.4 & 0.3 \\
86 & 15 & 0.12 & 0.48 & 0.5 & 0.3 \\
87 & 19 & 0.12 & 0.54 & 0.6 & 0.3 \\
88 & 23 & 0.12 & 0.58 & 0.6 & 0.3 \\
89 & 1 & 1.05 & 0.76 & 1.5 & 0.5 \\
90 & 1 & 1.05 & 1.27 & 1.9 & 0.4 \\
91 & 2 & 1.05 & 1.41 & 2.0 & 0.4 \\
92 & 2 & 1.05 & 1.53 & 2.1 & 0.3 \\
93 & 544488 & 0.0037 & 2.70 & 2.7 & 0.2 \\
94 & 0.000113 & 13.9 & 0.15 & 13.9 & $\mathrm{NA}$ \\
95 & 0.000113 & 806.0 & 8.54 & 805.6 & $\mathrm{NA}$ \\
96 & 0.000113 & 1028.0 & 10.90 & 1027.9 & $\mathrm{NA}$ \\
97 & 0.000113 & 1389.0 & 14.73 & 1389.0 & 0.1 \\
98 & 0.000113 & 2222.0 & 23.57 & 2222.5 & 0.3
\end{tabular}




\section{Appendix B: Governing Equations}

The scaling presented in Chapter 2 provides a highly simplified glimpse into the mathematical formulation of such free surface flows. Using the same scale parameters defined previously and preserving the aspect ratio, $\gamma=r_{o} / h_{b}$ we present the complete equations describing these axially separated liquid bridges to the point of rupture. The momentum equations in a $2-\mathrm{D}$ rotationally symmetric model are given as;

$z$-component of momentum

$$
\begin{aligned}
\frac{\rho r_{o} \hat{U}}{\sigma}\left(1+\frac{U_{o}}{\hat{U}}\right)^{2} & \left(\frac{\partial U_{z}}{\partial t}+U_{r} \frac{\partial U_{z}}{\partial r}+U_{z} \frac{\partial U_{z}}{\partial z}\right)= \\
& -\frac{\partial P}{\partial z}+\frac{\mu \hat{U}}{\sigma}\left(1+\frac{U_{o}}{\hat{U}}\right)\left(\gamma^{-1} \frac{1}{r} \frac{\partial}{\partial r}\left(r \frac{\partial U_{z}}{\partial r}\right)+\gamma \frac{\partial^{2} U_{z}}{\partial z^{2}}\right)+\gamma^{-1} \text { Bo }
\end{aligned}
$$

and $r$-component of momentum

$$
\begin{aligned}
\gamma \frac{\rho r_{o} \hat{U}}{\sigma}\left(1+\frac{U_{o}}{\hat{U}}\right)^{2} & \left(\frac{\partial U_{r}}{\partial t}+U_{r} \frac{\partial U_{r}}{\partial r}+U_{z} \frac{\partial U_{r}}{\partial z}\right)= \\
& -\gamma^{-1} \frac{\partial P}{\partial r}+\frac{\mu \hat{U}}{\sigma}\left(1+\frac{U_{o}}{\hat{U}}\right)\left(\frac{1}{r} \frac{\partial}{\partial r}\left(r \frac{\partial U_{r}}{\partial r}\right)+\gamma^{2} \frac{\partial^{2} U_{r}}{\partial z^{2}}-\frac{U_{r}}{r^{2}}\right)
\end{aligned}
$$

The continuity equation is given as

$$
\frac{1}{r} \frac{\partial}{\partial r}\left(r U_{r}\right)+\frac{\partial U_{z}}{\partial z}=0
$$


The kinematic boundary condition is given as

$$
U_{r}=\frac{\partial r}{\partial z} U_{z}+\frac{\partial r}{\partial t}
$$

The normal and tangential stress equations are given as

$$
\vec{n} \cdot \vec{T} \cdot \vec{n}+P_{o}=\sigma \kappa
$$

which becomes

$$
\frac{2 \gamma^{3}\left(\left(\frac{\partial r}{\partial z}\right)^{2} \frac{\partial U_{z}}{\partial z}-\frac{\partial r}{\partial z} \frac{\partial U_{r}}{\partial z}\right)-\gamma^{2}\left(\frac{\partial r}{\partial z}\right)^{2} P+2 \gamma\left(\frac{\partial_{r}}{\partial r}-\frac{\partial r}{\partial z} \frac{\partial U_{z}}{\partial r}\right)-P}{1+\gamma^{2}\left(\frac{\partial r}{\partial z}\right)^{2}}+P_{o}=
$$

and

$$
\vec{t} \cdot \vec{T} \cdot \vec{n}=\tau
$$


which becomes

$$
\frac{\gamma^{4}\left(\frac{\partial r}{\partial z}\right)^{2} \frac{\partial U_{r}}{\partial z}+\gamma^{2}\left(\frac{\partial U_{r}}{\partial z}+2 \frac{\partial r}{\partial z} \frac{\partial U_{r}}{\partial r}-\left(\frac{\partial r}{\partial z}\right)^{2} \frac{\partial U_{z}}{\partial r}-2 \frac{\partial r}{\partial z} \frac{\partial U_{z}}{\partial z}\right)}{1+\gamma^{2}\left(\frac{\partial r}{\partial z}\right)^{2}}-\frac{-\gamma\left(2 \frac{\partial r}{\partial z} P_{o}\right)+\frac{\partial U_{z}}{\partial r}}{1+\gamma^{2}\left(\frac{\partial r}{\partial z}\right)^{2}}=\tau .
$$

Note that both $\hat{U}$ and $U_{o} / \hat{U}$ are expressions based on the Suratman number $\mathrm{Su}=$ $\rho r_{o} \sigma / \mu^{2}$ and the instrument Reynolds number $\operatorname{Re}_{o}=\rho r_{o} U_{o} / \mu$. Both Suratman and Reynolds numbers may be written in terms of the instrument Weber and Capillary numbers, $\mathrm{Su}=\mathrm{We}_{o} / \mathrm{Ca}_{o}^{2}$ and $\mathrm{Re}_{o}=\mathrm{We}_{o} / \mathrm{Ca}_{o}$, and recalling that the limits of Eq. 2.9 evaluate to both the instrument Weber and Capillary numbers we see that the governing systems has a 3 term dependence $\left(\mathrm{We}_{o}, \mathrm{Ca}_{o}, \gamma\right)$ when gravity is neglected, Bo $\ll 1$. 